\title{
Competitive Effects of Requiring Sales Thresholds to Trigger Higher Commissions
}

\author{
Guillermo Gallego \\ Industrial Engineering and Operations Research, Columbia University \\ Masoud Talebian \\ School of Mathematical and Physical Studies, University of Newcastle
}

\begin{abstract}
We consider a game between two capacity providers that compete for customers through a broker who works on commissions and sells to both loyal and non-loyal customers. The capacity providers compete by selecting commission margins and sales thresholds at which commissions on all sales increase. We show that in equilibrium, contracts require positive sales thresholds. The threshold requirement can be best described as a mechanism for one provider to profit at the expense of the other. For exogenous commission margins, we show that it is the provider with the lower margin who benefits from thresholds at the expense of the broker. However, the gains for the lower margin provider can be a mirage in full equilibrium, where commission margins are endogenous.
\end{abstract}

Key words: Provider-Broker Competition, Contract Theory, Quantity Discount, Game Theory, Nash Equilibrium*

\section{Introduction}

Brokers play an important intermediary role between capacity providers and consumers and in some industries they are responsible for a large portion of sales. Providers rely on brokers because they are closer to customers, while customers prefer brokers because they view them as one stop shops where they can purchase products from different providers.

Capacity providers and brokers have different incentives resulting in decentralized management. Revenue splitting contracts between different business agents facilitate decentralized management. In this paper we study sales commission contracts, which are one of the most common contracts between capacity providers and brokers in the service industries. In this type of contract, sales occur through brokers who receive a commission from providers for each unit sold.

Our work is motivated by our observation of business contracts, where commission margins depend on sales volumes. A common practice is to impose minimum sales volumes to increase *We have greatly benefited from discussions with colleagues at Columbia University, and would like to specifically thank Jay Sethuraman and Ozge Sahin for their helpful comments. We are also grateful to the Department editor, Associate editor, and the reviewers for their helpful comments and suggestions for extending the model. 
commission margins. In other words, the broker is paid only a partial, often zero, commission margin unless sales exceed a set threshold. If sales exceed the threshold, the broker is paid the commission margin on all units, not just the units above the threshold. Sales thresholds may distort the effort that the broker exerts on non-loyal customers, as it may be optimal for the broker to steer demand to a provider to reach or exceed the threshold.

A practical motivation for our study is the relationship between competing service providers, e.g., airlines and hotels, which sell their capacity through travel agents, and have fixed capacity over the contracting horizon. Travel agents include, in addition to traditional brick-and-mortar stores, online travel agents (OTAs), such as Expedia, Orbitz and Travelocity. While in the US market, the commissions on simple domestic flights have vanished, they still are a major source of revenue for travel agents in other regions. Imposing sales targets is becoming a common practice in this industry. All 8 Asian airlines participating in a survey indicated that they use over-ride commission or back-end incentives. These remuneration programs offer agents bonus commissions if their sales exceed some target levels set by airlines. (Alamdari (2002))

In practice, multiple providers of different services, e.g., flight and hotel, interact with multiple brokers to sell their products. We focus on a stylized model to study the interactions between two competing capacity providers and a single broker. We hope the stylized model provide some insight into what the different players can expect as the outcome of introducing thresholds into commission contracts. .

In our setting, we concentrate on how thresholds and margins are decided when the broker can influence non-loyal customers to buy from either of the providers. On the basis of the providers' commission schedules, the broker decides how many units of product to sell from each provider. We show that in equilibrium providers impose positive thresholds, although this may harm them relative to the solution where thresholds are exogenously set at zero. We show that in our setting it is competition and not channel coordination that drives providers to set positive sales thresholds.

We also study the effect of the size of the market and the power of the broker to influence demand. We show that the broker always benefits from more power, but not necessarily from market growth; more explicitly, the broker may suffer from market growth. The reason for this is that when demand is abundant relative to capacity, the providers can pay low commission margins to generate sales. We also show that non-loyal demand coupled with relative large capacities results in high commission margins in equilibrium. This may help justify large broker margins in service industries with large capacities.

\subsection{Model Description and Assumptions}

Our model consists of two providers with fixed capacities under competition and one broker with access to customers' demand. This results in both horizontal competition between providers and 
vertical competition between providers and the broker. This setting allows us to study the effect that thresholds have on the way revenue is split among the providers and the broker.

We assume that products are partially substitutable; there are loyal customers who are interested in only one of the providers' products and non-loyal customers that can be influenced by the broker. The broker's power is measured by the ratio of non-loyal to total demand, and this ratio is assumed to be common knowledge to all players. The broker's power is a measure of the ability to influence customers to buy one product over the other, by allocation his sales effort. The demand can be affected by non-price incentives such as advertising, reward points, more information about one product, providing attractive shelf space, and guiding consumer purchases with sales personnel.

As products become closer substitutes, customers become more indifferent to the provider, making the broker's role more important in deciding which product to offer to non-loyal customers. In such settings, providers need to rely more on the broker to sell their products. In a commission contract, one common mechanism to persuade the broker to sell more of the provider's product is to impose sales thresholds to trigger commission margins.

Providers face a dilemma in setting sales thresholds. If a provider sets the thresholds too low or too high, she may lose sales as the broker's sales efforts may be directed toward the other provider. In equilibrium, the broker may be forced to buy more units than he can sell in order to obtain better terms, and then discard unsold units. Thus, the game is between providers, but must take into account how the broker allocates his sales efforts in response to thresholds and commission margins. We use Game Theory to analyze the players' interactions and the resulting equilibria on the basis of the players' sets of strategies.

While the game with endogenously determined margins and sales thresholds seems like the natural and more general setting for our study, our initial motivation came from service industries where commission margins had been fixed for a long time and were paid on all sales. In other words, travel agents operated in a world with fixed commissions without the need to meet sales volumes to earn them. As some service providers started experimenting with positive thresholds to trigger commissions, the commission margins still were considered fixed. This setting naturally raised interesting questions such as: when should providers impose sales thresholds? Who benefits and who loses from the introduction of thresholds? And are the benefits sustainable when margins are endogenous? Or are there reversals in fortunes by the introduction of sales thresholds that would render the long term tradition of fixed margins and zero thresholds a better solution for the different players? These are some of the questions that we can answer by studying the problem with exogenous and endogenous margins in the presence of thresholds. We study both settings and compare how revenue splits before and after the introduction of sales thresholds. This allows us 
to compare and contrast the effects of imposing sales thresholds with exogenous and endogenous margins

We assume that the prices of the products are fixed and exogenous since we are mainly interested in analyzing the players' interactions and how the revenue is split between them. We also assume that providers' capacities and the broker's power are exogenously determined and fixed. The fixed prices, capacities, and demand structure may be a result from competition at a higher strategic level, and are considered fixed during the contract designing.

We also consider a deterministic demand model to focus on strategic effects of requiring the thresholds on the profit split among the players. There exist a growing body of evidence in the literature, e.g., Gallego and Stefanescu (2009), which supports studying deterministic models to capture first order effects when considering strategic decisions. In this setting, we present a new justification for commission contracts with sales thresholds by showing that such contracts arise as the only equilibrium in competition. We show that our results are smooth to changes in demand so our results may be robust to demand mis-specifications.

We assume that the broker's and the providers' costs are negligible and they prefer to fulfill demand even if they do not make a profit. ${ }^{1}$ In the case of the broker, this can be justified when selling costs are negligible compared to the ill-will of unsatisfied customers. For providers, it can be justified since they have a sunk investment in capacity and we are assuming zero marginal fulfillment costs within capacity. In this setting when the fixed costs are sunk and variable production and distribution costs are negligible, maximizing the profit results in maximizing the revenue. Notice that the commissions are neither negligible nor fixed and providers maximize profits net of commissions paid to the broker, so we could use the term profit for the providers. However, we use the term revenue following the tradition of the revenue management literature to mean revenues net of commissions.

\subsection{Analogy between Commission Contracts and Sales Contracts}

Notice that a commission contract is similar to a sales contract, where a retailer buys the products from the supplier to sell them to customers. Requiring minimum sales volumes to trigger a commission increase is similar to all-units quantity discounts; the retailer receives a discount based on the size of his order. In this setting, the suppliers set a wholesale price and give discounts if the retailer reaches the set thresholds. Knowing the suppliers' quantity discount schedules, the retailer decides on optimal purchase quantities and determines sales that maximizes his revenue. The suppliers maximize their revenue by optimizing their discount schedules. The discount schedule affects how the supply chain's revenue is distributed among the players.

\footnotetext{
${ }^{1}$ In other words, we assume that the players do not have any reservation. If the players have a positive reservation, conditioned to be small enough, side payments can be set to satisfy it without changing the setting.
} 
Since our primary source of motivation is analyzing the service industry, where commission contracts are prevalent, for the rest of this paper we utilize the provider/broker terminology. However, because of this similarity some of our results and insights may apply to all-units quantity discount contracts and supplier/retailer relationship. The main difference is that in our setting we do not need to worry about inventory carrying costs.

From an economics perspective this research is related to the classical Bertrand model of oligopoly competition. Bertrand model considers competing providers that sell their products directly to the market and compete on offered prices to customers. In our setting, providers sell their products through a common broker and compete on offered commission margins to the broker. In other words, Bertrand model studies business-to-customers relationship, while we study a parallel model in business-to-business relationship.

The original Bertrand model does not consider the capacity constraints. In the BertrandEdgeworth model, the Bertrand model is generalized by considering cases where firms have capacity constraints, or more generally the marginal production cost increases as capacity increases. Without capacity constraints, competition drives down the prices to marginal costs. Our model extends the Bertrand-Edgeworth model in several directions. First, we investigate a supply chain, where the providers are in indirect contact with the market through a broker. Second, we consider a more general form of wholesale price contract. And third, we consider products which are partially substitutable, while the original Bertrand model assumes that the products are identical and all customers buy the product with the lowest price.

The rest of the paper is organized as follows. After a review of the supply chain coordination literature in Section 2, we provide variables definition and model formulation in Section 3. In Section 4, we analyze the effects of requiring sales thresholds to trigger a commission increase. In the last section, we summarize our findings and provide conclusions and avenues for further research.

\section{Literature Review}

There is much evidence in theory and practice that shows supply chains are not necessarily coordinated, so the supply chain's profit in a decentralized system is less than the optimal profit in a centralized system. ${ }^{2}$ This situation puts all members in a moral hazard situation because seeking their own profit costs other members. There are two streams of literature which focus on this subject: contract theory in economics and supply chain coordination in operations management.

\footnotetext{
${ }^{2}$ Interestingly, the incoordination can also exist in a single organization when different decisions are made separately. For example, Koabiyikoglu et al. (2010) consider price decisions and availability decisions and compare the performance of hierarchical models versus coordinated models.
} 
Tirole (1988) and Spengler (1950) provide an initial identification and review of supply chains coordination failure in the economics and operations management literature, respectively. Under a setting where the supplier sets a linear wholesale price, double marginalization and low stocking are the major factors that can make a chain uncoordinated.

One of the initial studies about double marginalization is by Jeuland and Shugan (1983). They consider a deterministic demand rate that is a decreasing function of the retail price. The supplier chooses the wholesale price and the retailer chooses the retail price. They show that this simple setting results in a retail price that is higher than the chain optimal one. This analysis confirms the previous studies' conclusions, e.g., Cournot (1838), that lack of coordination results in higher prices paid by customers. Jeuland and Shugan (1983) extend this incoordination problem to vertical chains with at least two members. However, they find it complicated to extend the model to multiple suppliers or multiple retailers when they are competing with each other.

Lariviere (1999) considers a news-vendor setting where the demand is stochastic, but the retail price is exogenous. In this setting, it can be shown that the retailer orders less than the chain's optimal amount so the double marginalization problem manifest itself through low stocking levels

While one possible solution to coordination problem is vertical integration and joint ownership, as Klein et al. (1978) propose, in many situations this solution is not feasible. Another solution is that the upstream supplier makes retailer's operational decisions. For example, in vendor-managed inventory (VMI) settings, inventory decisions are made by the supplier; refer to Fry et al. (2001) for a more detailed discussion about a common type of VMI agreements and some industry examples. A coordination tool which does not rely on contracts is collaborative planning and forecasting between chain's members; for example, we refer to Aviv (2001).

Another approach to increase coordination in a supply chain is using more sophisticated contracts between business agents, which is becoming more common and are the focus of our paper. The extensive literature on supply chain coordination is surveyed by Cachon (2003). Some of the most widely used supply chain contracts are described in Table 1 and they all coordinate a news-vendor chain.

Hax and Candea (1984) investigate cases where incremental quantity discount is equivalent to two-part tariff. Weng (1995) considers a setting in which fixed ordering costs affect order quantity and as a result inventory and operating cost and drives to an uncoordinated chain. He shows that two types of quantity discount contracts, all-units and incremental, perform identically in presence of fixed ordering costs. Moreover, he shows how quantity discounts and franchise fees can coordinate a chain with fixed ordering costs. In addition to achieving coordination, quantity discounts also can be used for price discrimination, see Kinter (1970) for more discussion. 
Table 1 Widely used contracts to coordinate a news-vendor chain

\begin{tabular}{|c|c|}
\hline Contract & Description \\
\hline $\begin{array}{ll}\text { All-units } & \text { quantity } \\
\text { discount } & \end{array}$ & The supplier charges a volume-depended per unit price to all purchased units. \\
\hline $\begin{array}{l}\text { Incremental quan- } \\
\text { tity discount }\end{array}$ & $\begin{array}{l}\text { The supplier charges a volume-depended per unit price just to units above a } \\
\text { threshold and a higher wholesale price to the rest of the units. }\end{array}$ \\
\hline Revenue sharing & The retailer pays a wholesale price and a share of revenue to the supplier. \\
\hline $\begin{array}{l}\text { Full return } \\
\text { (Buy-back) }\end{array}$ & $\begin{array}{l}\text { The supplier charges a wholesale price per unit, but the retailer returns the } \\
\text { unsold units to the supplier at the end of the sales period for a predetermined } \\
\text { amount per unit. }\end{array}$ \\
\hline $\begin{array}{l}\text { Sale rebate } \\
\text { (Target rebate) }\end{array}$ & $\begin{array}{l}\text { The supplier charges the retailer a per unit wholesale price, but pays the } \\
\text { retailer a rebate per unit sold above a fixed target and the retailer continues } \\
\text { to salvage leftover units. }\end{array}$ \\
\hline
\end{tabular}

As it can be observed, most of the current coordination literature focuses on the monopoly case, or non-competing multiple suppliers and/or retailers. There are a limited number of studies that consider these contracts under suppliers' competition. Heese (2008) examines interactions in a supply chain when two suppliers are competing for product sales through a common retailer. However, in this setting, the retailer profits from the selling of extended warranties, not the suppliers' units of product. Therefore, he also is not considering the suppliers' competition through the retailer. Mahajan and van Ryzin (2001) consider competition among several suppliers that indirectly influence customer's choice through inventory policy. They show that the equilibrium inventory levels are higher than joint optimal levels. However, they do not investigate other types of contracts rather than wholesale price. Boyaci and Gallego (2004) study two competing supply chains which compete for a higher market share via customer service. They show that coordination is a dominant strategy for both supply chains, but it does not necessarily increase their profit. However, they do not investigate the contracts that can coordinate the chain and also do not allow retailers to buy from more than one supplier.

As a brief review of the literature has shown, sophisticated contracts are usually recommended to increase the total revenue of a supply chain, i.e., coordination. However, we focus our attention on how supply chain revenues are split in a competition assuming fixed exogenous selling prices and sales effort, zero marginal fulfillment costs within capacity, and deterministic demands. By these assumptions, we avoid traditional causes of incoordination, i.e., double marginalization, low stocking, and low sales effort.

One of the closest works to our paper is Cachon and Kok (2010). They analyze the effect of two-part tariff and a subset of incremental quantity discount and compare them to wholesale price 
with price sensitive customers. We focus on a different contract, all-units quantity discount and in addition, we consider suppliers with limited capacity. Nevertheless, they also reach the conclusion that more sophisticated contracts are not necessarily beneficial for suppliers in equilibrium.

It should be noted that quantity discount contracts and their terms, like all other discussed contracts, have litigation aspects too. Tirole (1988) discusses that only verifiable parameters should be written into a contract so that in the case of a disagreement between the contracting parties, a court can intervene. This implies that just observation by both parties is not enough and the parties should be able to prove their observations.

\section{Formulations}

In this section, we present the formulations for each of the players. We have two finite capacity providers and a broker who plays the intermediary role between providers and the market. The market consists of loyal and non-loyal customers; non-loyal customers can be assigned by the broker to any of the providers. Each player's formulation is discussed after defining the following parameters and decision variables.

Parameters:

- $c_{i}$ : Capacity of provider $i$.

- $p_{i}$ : Price of provider $i$ 's product.

- $d_{i}$ : Loyal demand for provider $i$ 's product.

- $d_{0}$ : Non-loyal demand which the broker can tilt.

Providers' decision variables:

- $0 \leq m_{i} \leq p$ : The full commission margin that provider $i$ pays the broker on all units if sales exceed the threshold $t_{i}$.

- $0 \leq t_{i} \leq c_{i}$ : The sales threshold required by provider $i$ to obtain the full commission margin $m_{i}$ on all units sold.

The broker's decision variables:

- $s_{i}$ : Units from provider $i$ sold by the broker, $i=1,2$.

Figure 1 shows the model scheme, which is common knowledge to all players. The broker's power can be measured by the relative size of the non-loyal to the total demand and represents the proportion of the customers that can be influenced by the broker. In our model, the providers differ in their capacity $\left(c_{i}\right)$, their loyal market $\left(d_{i}\right)$, and the price they charge for their products $\left(p_{i}\right)$.

The strategy space for the providers consist of the pair $\left(t_{i}, m_{i}\right)$ where $t_{i}$ denotes the threshold and $m_{i} \in\left[0, p_{i}\right]$ is the per unit commission margin that the broker gets from selling above the threshold $t_{i}$ from provider $i$. The broker obtains no commission from provider $i$ if he sells fewer 


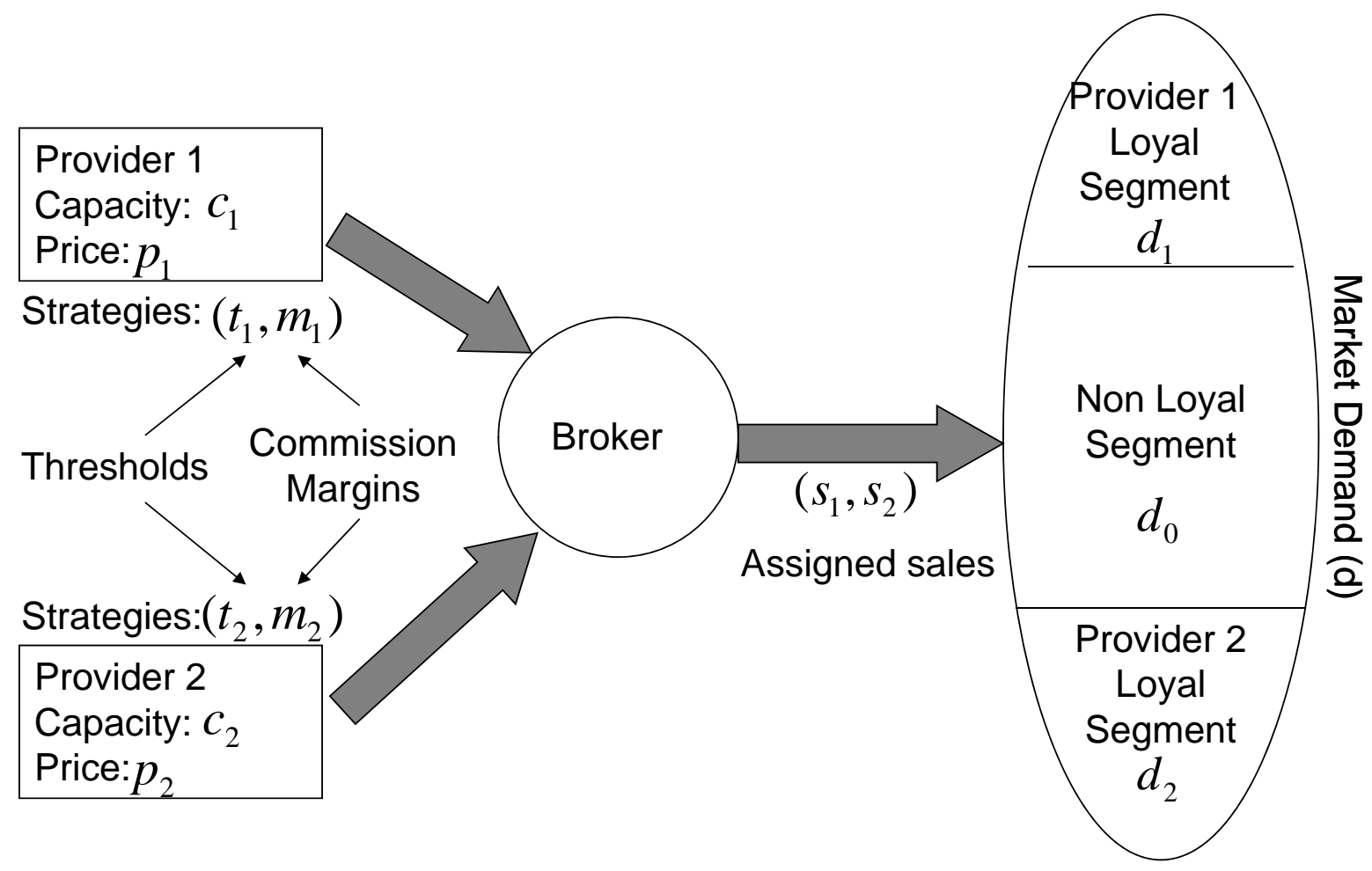

Figure 1 Game Scheme

than $t_{i}$ units and obtains commission $m_{i} s_{i}$ if he sells $s_{i} \geq t_{i}$ units from provider $i=1,2$. Notice that the commission scheduled is described by only two parameters: the threshold $t_{i}$ and the commission margin $m_{i} \in\left[0, p_{i}\right]$ that operates on sales $s_{i} \geq t_{i}$. While this commission schedule may seem very limited, it is possible to show (see Theorem 1 below) that a provider cannot do better by considering more general commission schedules, such as all unit schedules with multiple thresholds or incremental quantity commission schedules.

The strategy of the broker is to assign sales of the non-loyal segment $d_{0}$ among the providers to maximize his profits from commissions. In principle, the broker's strategy may involve purchasing units beyond those he can sell (discarding) with the purpose of achieving sales thresholds. Theorem 1 below shows that the broker can limit his strategy, without loss of generality, to avoid discarding. Theorem 1, therefore justifies the reduction from a model with general commission schedules and discarding to our two-parameter commission structure and no discarding.

THEOREM 1. We can assume, without loss of generality, that:

- For every non-negative and non-decreasing commission schedule, there exists a two parameter schedule $\left(t_{i}, m_{i}\right)$ that results in the same sales and revenue split between providers and the broker.

- For every equilibrium that allows discarding, there exists an equilibrium without discarding resulting in the same revenue split between providers and the broker. 
Theorem 1 simplifies the analysis because we only need to consider two parameter policies for each provider instead of having to work with general commission schedules and potential discarding of units purchased just for the purpose of reaching a threshold. The proof of Theorem 1 is quite involved and can be found in the Appendix.

Definition 1. Define

- $b_{i}=\min \left[c_{i}, d_{0}+d_{i}\right]$

- $r_{i}=\min \left[b_{i}, d-b_{j}\right]$

Notice that $b_{i}$, as the minimum of the capacity and the maximum demand that can be channeled to provider $i$, equals the maximum sales of provider $i$, and $r_{i}$, as the minimum of the capacity and the residual demand, equals the minimum sales of provider $i$. One can also think of $b_{i}$ and $r_{i}$ as sales associated with getting first dibs or second dibs on the market. More precisely, $b_{i}$ is the amount that provider $i$ would sell if the broker gave him priority over provider $j$. Conversely, $r_{i}$ is the amount that provider $i$ would sell if the broker gave priority to provider $j$. We also refer to $b_{i}$ as the sellable capacity of provider $i$.

Since each provider sells at least $r_{i}$, the only nontrivial thresholds are $t_{i} \geq r_{i}, i=1,2$. The case of zero thresholds, $t_{i}=0 i=1,2$, will refer to the common situation where sales thresholds are not imposed. We refer to this case of inactive thresholds as "without thresholds", compared to the case of "with thresholds", which means that sales thresholds are decision variables selected by the providers.

Since the broker makes decision on the basis of the providers' decisions, we can and will reduce the game to a duopoly after accounting for the broker's behavior. In this duopoly, the providers are the two players and the broker's optimal policy determines their payoff functions.

\subsection{Broker}

The broker, as an intermediary between providers and the market, can influence customers' choice by deciding how to distribute sales efforts and marketing capabilities between providers' products.

The broker's power is measured by the size of the non-loyal demand $d_{0}$ relative to the total demand $d=d_{0}+d_{1}+d_{2}$. Extremes of $d_{0}=0$ and $d_{0}=d$ represent a powerless and fully powerful broker respectively. As $d_{0} / d \in[0,1]$ increases from zero to one, competition between providers becomes more severe.

Let $\pi_{B}$ denote the broker's revenue, the broker's formulation with nontrivial thresholds is given by:

$$
\begin{gathered}
\pi_{B}\left(t_{i}, m_{i}\right)=\max _{\left(s_{i}, k_{i}\right)} k_{1} m_{1} s_{1}+k_{2} m_{2} s_{2} \\
r_{i} \leq s_{i} \leq b_{i}
\end{gathered}
$$$$
\text { for } i=1,2
$$ 


$$
\begin{array}{ll}
k_{i} t_{i} \leq s_{i} & \text { for } i=1,2 \\
s_{1}+s_{2} \leq d & \\
k_{i} \in\{0,1\} & \text { for } i=1,2 .
\end{array}
$$

In this formulation, $k_{i}$ is a binary variable that determines whether or not the provider $i$ ' threshold is reached by the broker. If the broker does not reach the threshold, corresponding to $k_{i}=0$, provider $i$ does not pay a commission margin, getting a free ride. Note that in the broker's formulation, the broker decides what part of the non-loyal demand should be assigned to each of the providers. For each solution $s_{i}$, the broker assigns $\left(s_{i}-d_{i}\right)^{+}$of $d_{0}$ to provider $i$.

\subsection{Providers}

In the formulation and analysis, we set $j=3-i$ without further notice to refer to the provider competing with provider $i=1,2$. Let $\pi_{i}\left(t_{j}, m_{j}\right)$ denote the optimal revenue of provider $i$ as a function of $\left(t_{j}, m_{j}\right)$. Then,

$$
\begin{aligned}
\pi_{i}\left(t_{j}, m_{j}\right)= & \max _{\left(t_{i}, m_{i}\right)}\left(p_{i}-k_{i} m_{i}\right) s_{i} \\
& r_{i} \leq t_{i} \leq c_{i} \\
& 0 \leq m_{i} \leq p_{i}
\end{aligned}
$$

As it has been modeled, the $k$ 's and the $s$ 's are external variables for the providers and functions of $t$ 's and $m$ 's, so providers can influence them by their decisions rather than directly setting them. The fact that the objective functions are discontinuous and non-monotone in the strategies as a result of the binary threshold conditions makes some classical results from standard game theory not applicable. ${ }^{3}$

\subsection{Demand}

In our analysis in the next sections, we also discuss the effects of changes in the market size, $d$. For this purpose, we do not need to limit ourself to any specific demand model. We only require that the demand model satisfies the assumption that "as the market size, $d$, increases, $d_{0}$ and $d_{i}$ 's

\footnotetext{
${ }^{3}$ In the model, the thresholds have been capped by the capacities. It can be argued that thresholds should be capped by market demand, as it is not reasonable to put thresholds higher than market size. However, our model allows for scenarios where the providers put thresholds higher than the market size and induce the broker to buy and then discard unsold items, a behavior which we have anecdotal evidence of happening in practice. In some cases, it is not possible for the broker to discard extra units of the product to reach the thresholds if providers monitor sales and do not allow discarding. In this case, the broker is in a stronger position to argue that the thresholds should not exceed the market size. Without discarding the broker's response set is more limited and one can expect he should be worse off and the providers be better off. However, as a counterintuitive result, it is possible to show that the providers's revenues stay fixed or decreases. For an analysis of this scenario, refer to Talebian (2010).
} 
increase proportionally." As a result, the broker's power, represented by $\frac{d_{0}}{d}$, remains fixed as the demand scales up. This assumption is not restrictive and makes it possible to study the effect of the market size independent of the broker's power. We discuss multinomial logit (MNL) choice and market segmentation as two models that satisfy our assumption.

One way to model customers' behavior in choosing between different products is through the multinomial logit (MNL) choice framework. As Talluri and van Ryzin (2004) state MNL formulation models the real world closely, is analytically tractable, and is easy to estimate. Scaling all utilities based on customer elasticity, we define

- $u_{i}$ : The utility of each provider's product

- $v_{i}$ : The positive non-monetary utility added by the broker to the product of provider $i=1,2$.

- $v$ : The broker's ability to affect the utility of customers which is the bound on the utility that can be added by the broker $\left(v_{1}+v_{2} \leq v\right)$.

- $d_{i}\left(v_{1}, v_{2}\right)$ : Demand for each provider's product, conditioned that the broker assigns added utility $v_{i}$ to each provider.

We have:

$$
d_{i}\left(v_{1}, v_{2}\right)=\frac{\exp \left(u_{i}-p+v_{i}\right)}{\exp \left(u_{1}-p+v_{1}\right)+\exp \left(u_{2}-p+v_{2}\right)} d \text { for } i=1,2
$$

We can represent the loyal demand for provider $i$ as her minimal demand when all the effort is assigned to the other provider. ${ }^{4}$ Notice that in this model the broker's power is equal to $(d-$ $\left.d_{1}(0, v)-d_{2}(v, 0)\right) / d$, which increases by the $v$, and $v=\infty$ represents an extremely powerful broker.

Another way to model customers' behavior is market segmentation, where we define

- $\alpha$ : Non-loyal segment of the market

- $\beta_{i}$ : Each provider's share of loyal segment of the market $\left(\beta_{1}+\beta_{2}=1\right)$

Let:

$$
d_{i}=\beta_{i}(1-\alpha) d \quad \text { for } i=1,2
$$

Notice that the in this model, the broker's power is equal $\alpha$, and $\alpha=1$ represents an extremely powerful broker.

\section{Analysis}

We will start our analysis by disposing of the case when $b_{1}+b_{2} \leq d$. In this case there exists a unique pure-strategy equilibrium such that $m_{i}=0$ for $i=1,2$, which results in sales $s_{i}=b_{i}$ and revenue split $\pi_{i}=p_{i} b_{i}$ and $\pi_{B}=0$. In this setting, the market is so large that there is no competition

\footnotetext{
${ }^{4}$ Note that w.l.o.g., we can assume that the broker exerts all his power, which is fixed exogenously. Also notice that the broker's power does not notice the overall demand. Thus, for any $\left(v_{1}, v_{2}\right)$ such that $v_{1}+v_{2}<v$, there exists a $\left(v_{1}^{\prime}, v_{2}^{\prime}\right)$ such that $v_{1}^{\prime}+v_{2}^{\prime}=v$ and $d_{i}\left(v_{1}, v_{2}\right)=d_{i}\left(v_{1}^{\prime}, v_{2}^{\prime}\right)$ for $i=1,2$
} 
between providers, so commissions are zero and thresholds are irrelevant. As the market demand increases, the total market revenue increases, but it does not change the broker's revenue, which stays at zero. The broker's revenue is also insensitive to the broker's power, but the revenues for both providers are non-decreasing in the broker's power. The above analysis shows that the only nontrivial case is $b_{1}+b_{2}>d$, where the collective capacity is larger than demand, so the providers need to compete for the non-loyal customers. We assume from now on that this condition holds and give this a formal definition:

DeFinition 2. We call a market competitive if $b_{1}+b_{2}>d$.

Notice that the competitive condition is equivalent to $c_{i}>d_{i}$ for $i=1,2$ and $c_{1}+c_{2}>d$. It is also easy to see that in a competitive market $r_{i}=\min \left(b_{i}, d-b_{j}\right)=d-b_{j}$ for $i=1,2$. As a result, if provider $i$ is given priority then sales for the providers are $b_{i}$ and $d-b_{j}$, respectively for provider $i$ and $j$. There exists competition among the three players to collect a larger share of the total market revenue. The next two subsections deal with the competitive market problem without and with thresholds.

\subsection{Without Thresholds}

In this subsection we assume thresholds are set to $t_{i}=0$, and consequently the providers compete only with full margins. The providers choose commission margins that maximize their net revenues and the broker always give priority to the provider with the largest margin. Through this section, we label the provider with higher $\frac{b_{i}}{p_{i}}$, provider 1 . A higher capacity and/or market loyal share and a lower price are criteria for determining provider 1 . We use superscript "o" to refer to without threshold case.

Theorem 2. If $b_{1}+b_{2}>d$, there exists a mixed-strategy equilibrium such that for $m \in$ $\left[0, \frac{b_{1}+b_{2}-d}{b_{1}} p_{1}\right]: P\left(m_{i}^{o} \leq m\right)=\left(\frac{1}{p_{j}-m}\right)\left[\frac{\left(p_{j} b_{i}-p_{i} b_{j}\right)^{+}}{b_{i}}+\frac{m\left(d-b_{i}\right)}{b_{1}+b_{2}-d}\right]$ for $i=1,2$. Moreover, it results in revenue split:

$$
\begin{aligned}
& \pi_{i}^{o}=p_{i}\left(d-b_{j}\right)+\left(p_{i}-\frac{b_{i}}{b_{j}} p_{j}\right)^{+}\left(b_{1}+b_{2}-d\right) \text { for } i=1,2 \\
& \pi_{B}^{o}=p \frac{b_{2}}{b_{1}}\left(b_{1}+b_{2}-d\right) \text { if } p_{1}=p_{2}=p \\
& \pi_{B}^{o}=p_{1} b_{2}+\frac{p_{1}}{p_{2}-p_{1}}\left(d-b_{2}\right)\left(p_{2} \frac{b_{2}}{b_{1}+b_{2}-d}-p_{1} \frac{b_{2}}{b_{1}}\right) \ln \left(\frac{p_{2}\left(d-b_{2}\right)}{b_{1} p_{2}-\left(b_{1}+b_{2}-d\right) p_{1}}\right) \text { if } p_{1} \neq p_{2}
\end{aligned}
$$

Theorem 2 states that if the market is competitive, then providers will randomize their strategy and in every case, the broker prioritizes the provider who draws the largest margin. Notice that there exists a mass $\frac{1}{p_{2}}\left(\frac{p_{2} b_{1}-p_{1} b_{2}}{b_{1}}\right)$ at $m_{1}^{o}=0$ for provider 1 .

When capacity constraints are not binding and there are no loyal customers, i.e., $c_{1}+c_{2}>d=d_{0}$, we have a Bertrand model where the broker plays the intermediary role between providers and the 
market. In this case, provider 1 pays a commission equal to $p_{1}$ and does not gain any profit, as in the classical Bertrand result, while provider 2 gains a profit equal to $\left(p_{2}-p_{1}\right) d$.

Theorem 2 confirms that market demand growth results in more revenue for providers, but the revenues for the broker first increase and then decrease with the market size. The intuition is that an increase in aggregate demand can reduce the broker's revenues as the providers have less of an incentive to compete. Figure 2 shows each player's revenue as a function of market demand, when demand is not loyal. In this setting, provider 1 has a lower price but a higher capacity.

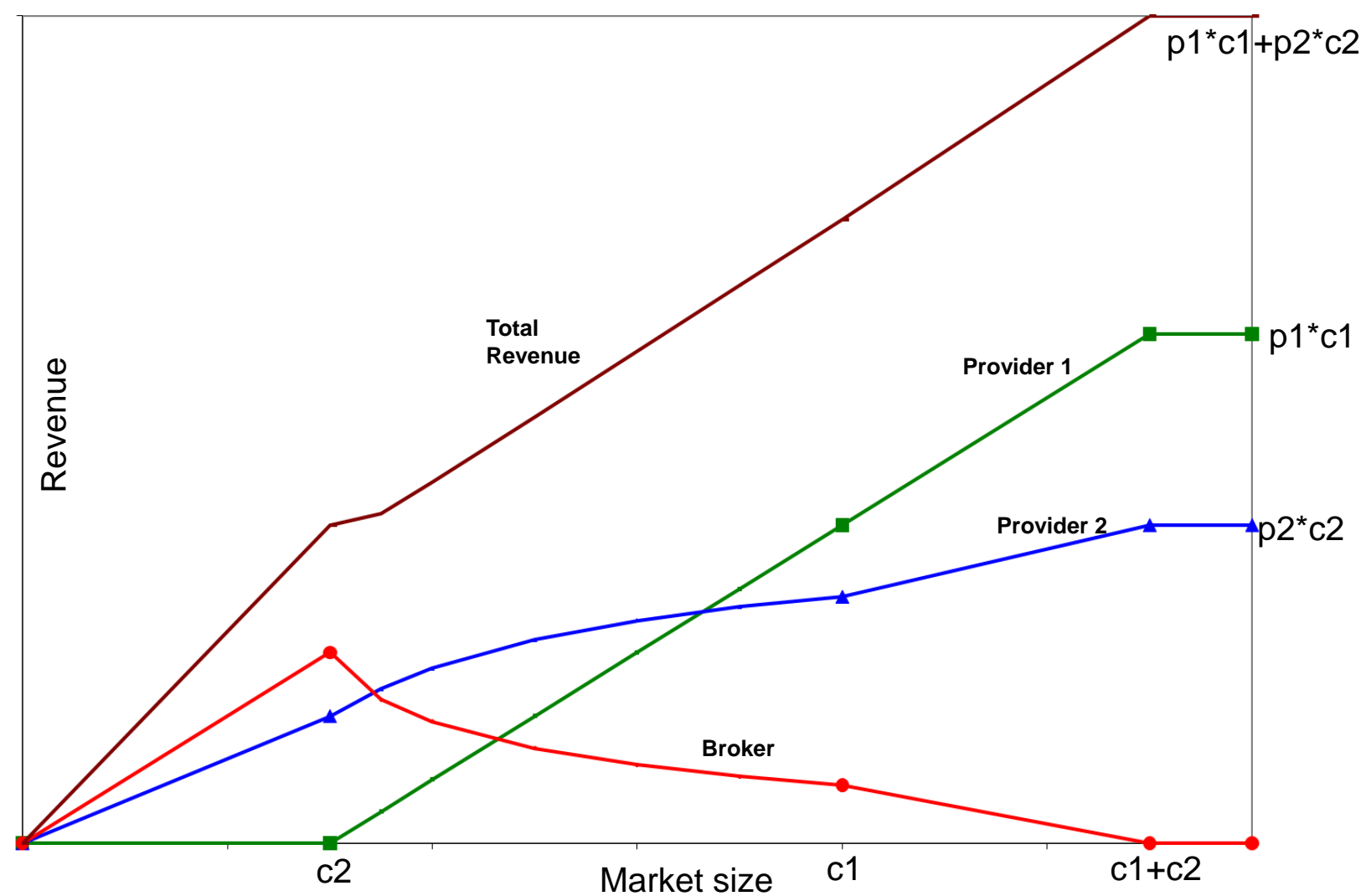

Figure 2 Non-loyal market without thresholds, $c_{1}=8, p_{1}=3, c_{2}=3, p_{2}=5$

Regarding the broker's power growth, as the broker power increases the broker gains, as illustrated in Figure 3, at the expense of the providers.

\subsection{With Thresholds}

Based on Theorem 1, we can and do limit our study to zero partial commission margins so each provider has a two-dimensional strategy to set: $\left(t_{i}, m_{i}\right)$. On the basis of Theorem 1 , we also can limit our study to $r_{i} \leq t_{i} \leq b_{i}, i=1,2$. Indeed, if $b_{i}<t_{i}$ the broker can decide to reach the threshold, $t_{i}$, which results in discarding from provider $i$ and on the basis of Theorem 1 , we can avoid studying 


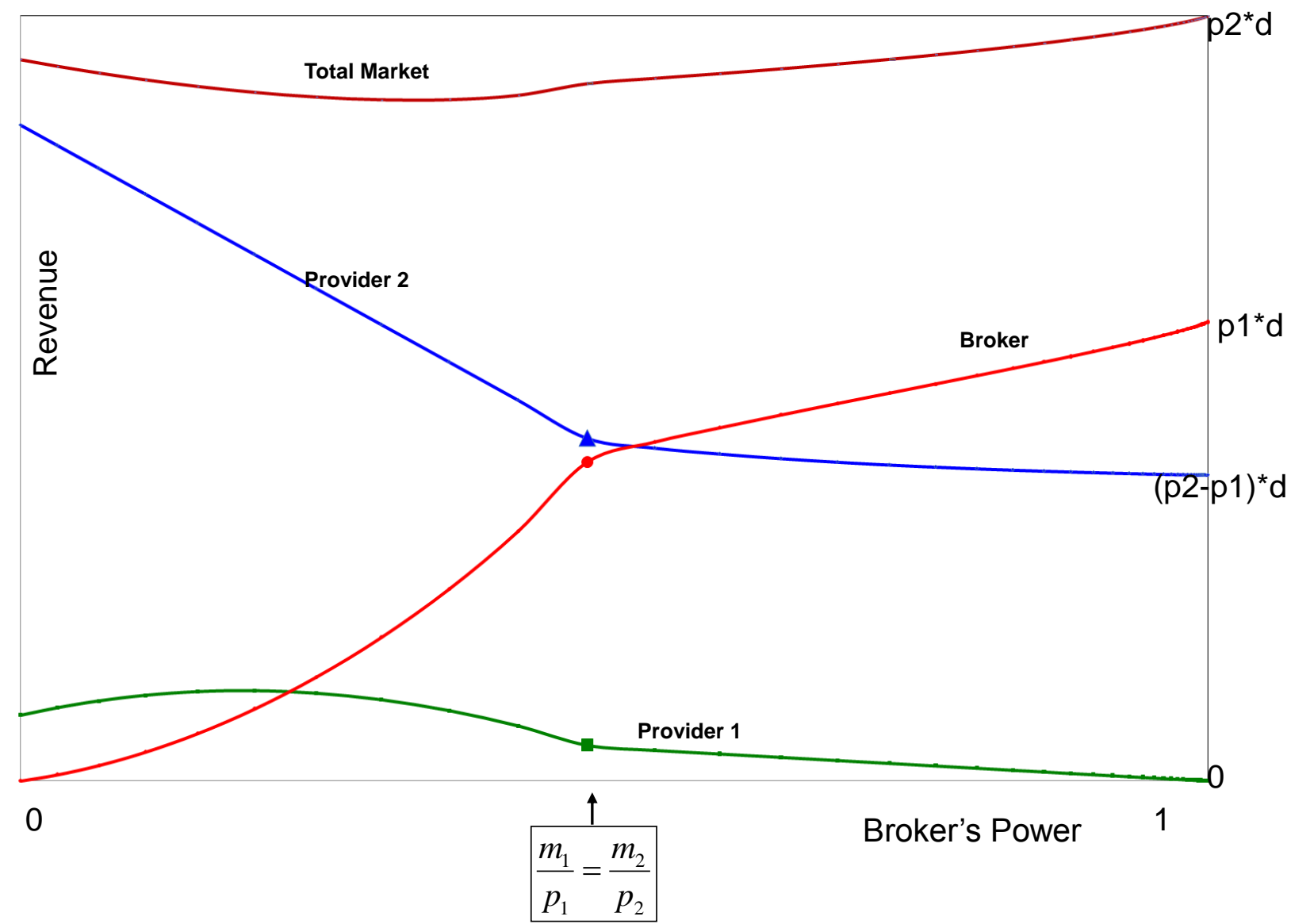

Figure 3 Large capacities without thresholds, $p_{1}=5, p_{2}=3, d=11$

this case. Alternatively, the broker can decide not to reach the threshold which results in free ride. This case can be captured by setting $m_{i}=0$.

Theorem 3. If $b_{1}+b_{2}>d$, there exists a pure-strategy equilibrium such that $m_{i}^{*}=$ $\frac{b_{1}+b_{2}-d}{b_{i}} \min \left[p_{1}, p_{2}\right]$ and $t_{i}^{*}=b_{i}$ for $i=1,2$. The equilibrium results in revenue split:

$$
\begin{array}{r}
\pi_{i}^{*}=p_{i}\left(d-b_{j}\right)+\left(p_{i}-p_{j}\right)^{+}\left(b_{1}+b_{2}-d\right) \text { for } i=1,2 \\
\pi_{B}^{*}=\min \left[p_{1}, p_{2}\right]\left(b_{1}+b_{2}-d\right)
\end{array}
$$

Calling the provider with higher price, primary provider, and labeling her provider $1, s_{1}^{*}=b_{1}$ and $s_{2}^{*}=r_{2}=d-b_{1}$. Notice that the provider 1 gets the first claim of the market. Also, notice that since the market is competitive and therefore $b_{2}>d-b_{1}$, provider 2 gets a free ride so the broker gets all his profits from provider 1. By comparing Theorems 2 and 3, one can compare the revenue of three players without thresholds and with thresholds. We postpone this discussion to Section 4.3 , where we discuss the strategic effects of sales thresholds.

Theorem 3 also provides insights into push vs. pull systems. In a pull system, customers already have chosen their brand and come to the broker to buy it, while in a push system, it is the broker 
who persuades customers and influences their choice. Our results confirm the general intuition that while in a pull system, commissions can be low; a push system requires high commissions to incentivize the broker. In practice, systems are usually a mix of push and pull systems, and the commission percentage depends on the mixture. The mixture between push and pull is related to the broker's power; $d_{0} / d$. As the broker's power increases, conditioned on existing enough capacity, the system moves toward a push system. The type of the system also depends on capacity versus demand; if capacity of provider $i$ is binding such that $d_{0}+d_{i}>c_{i}$, then as $c_{i}$ increases both commission margins increase and the system moves toward a push system. If capacity is already abundant, no change is expected. The managerial implication would be that a provider would set up a push or pull system depending on the market structure, size of her capacity, and also the competing provider's capacity.

Comparative statics based on Theorem 3 show that changes in providers' and the broker's profits as a result of demand changes are linear and capped by prices. It shows that the equilibrium solution is a smooth function of the problem parameters and there are no jumps. In other words, if the demand randomness compared to its size is small, the changes in profits are insignificant.

In addition, it shows that market growth always results in more revenue for the providers, but it can increase and then decrease the broker's revenue. Figures 4 shows how revenue splits between providers and the broker as market demand increases.

Figure 5 shows how revenue splits between the providers and the broker as broker power increases. It confirms that the broker's power positively affects the broker and negatively affects the providers when the market is competitive. This is in sharp contrast to the non-competitive case where surprisingly market power actually benefits the providers.

Providers may consider establishing direct sales channels to avoid paying commissions to the broker. While direct sales can change the market structure, we focus here on the hypothetical case where providers cannot reach the non-loyal segment through direct sales and the size of the loyal market segment is fixed. Under this assumption, the incentive to open a direct sales channel is to avoid paying commission on the loyal part of the demand. While this may seem a compelling motive, we show that the providers cannot benefit from offering direct sales. Let $\pi_{i}^{d}$ be the expected profit to provider $i$ assuming that loyal demand is sold through a direct sales channel.

TheOREM 4. $\pi_{i}^{d}=\pi_{i}^{*}$ for $i=1,2$.

Theorem 4 states that providers cannot benefit from direct sales in our hypothetical case. The intuition here is that when a provider starts to sell directly to loyal customers, based on Theorem 3, she needs to offer higher commission margins and lower sales thresholds to incentivize the broker. In other words, selling directly to the loyal market puts the provider in a weaker position to compete 


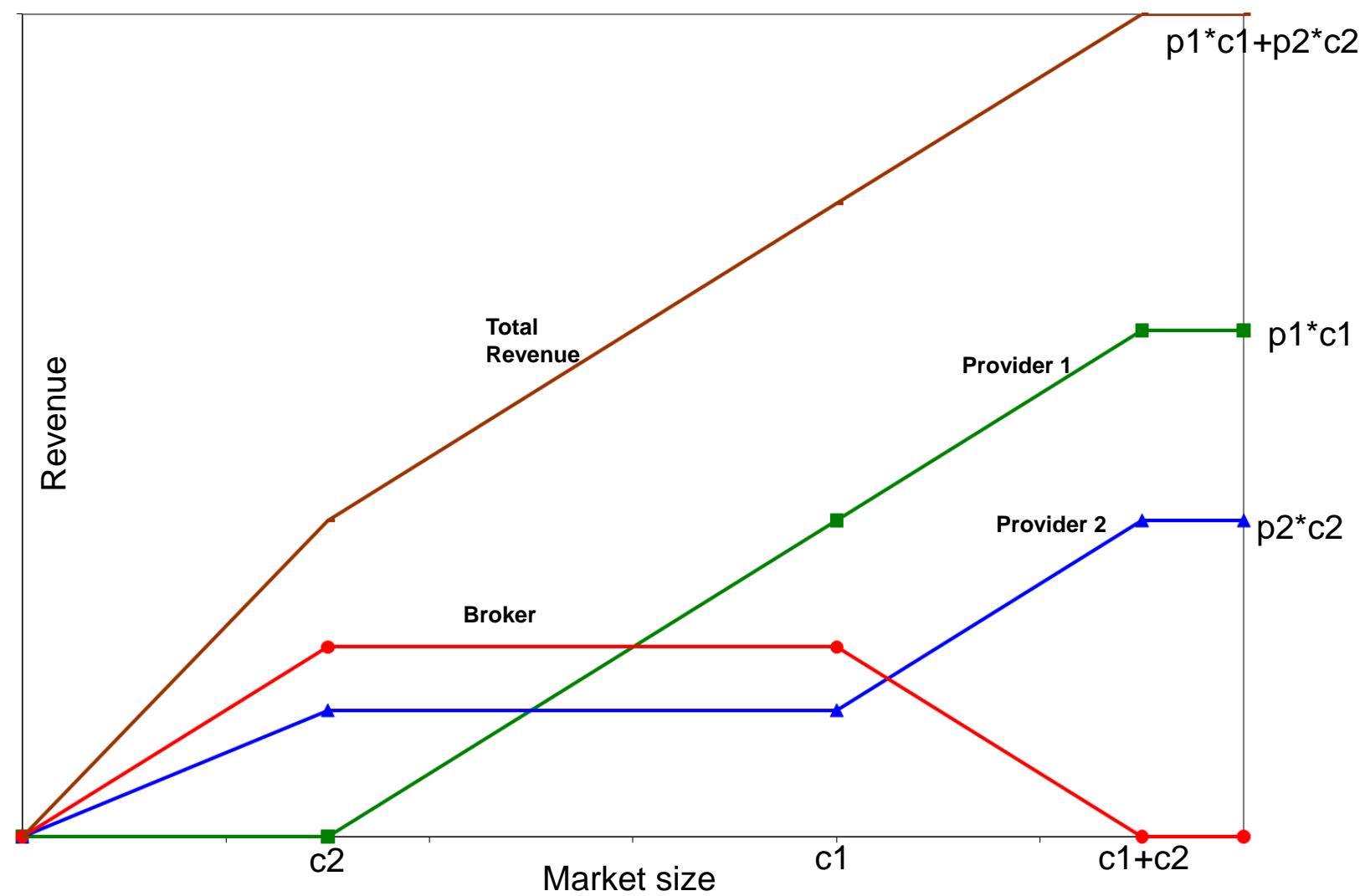

Figure 4 Non-loyal market with thresholds, $c_{1}=8, p_{1}=3, c_{2}=3, p_{2}=5$

for the non-loyal market, and savings of not paying commissions on the loyal demand are lost to the higher commissions paid on the non-loyal part. The result is independent of the the market structure, i.e., the relative size of loyal and non-loyal market.

The implication of Theorem 4 is that a hybrid business model that sells directly to loyal customers and via the broker to non-loyal customers can only be profitable for the provider if the direct sales channel can attract non-loyal customers. Considering the fact that in practice there are some costs associated with selling directly to loyal customers, Theorem 4 suggests that providers may be actually better off without direct sales channels unless they can significantly grow market share. In other words, there is a need to substantially increase the size of the loyal market through the direct channel to justify the dual channel strategy. It is interesting to notice that this result is based on the revenue distribution with thresholds. Without thresholds, providers are not indifferent between the hybrid model and selling only via the broker. By Theorem 2, provider 2 prefers direct sale.

\subsection{Strategic Effects}

In this section, we study the strategic effects of requiring sales thresholds. First, we notice that in presence of thresholds, a pure-strategy equilibrium exists, while without thresholds, there is no pure-strategy equilibrium and providers randomize their commission margins. If the market 


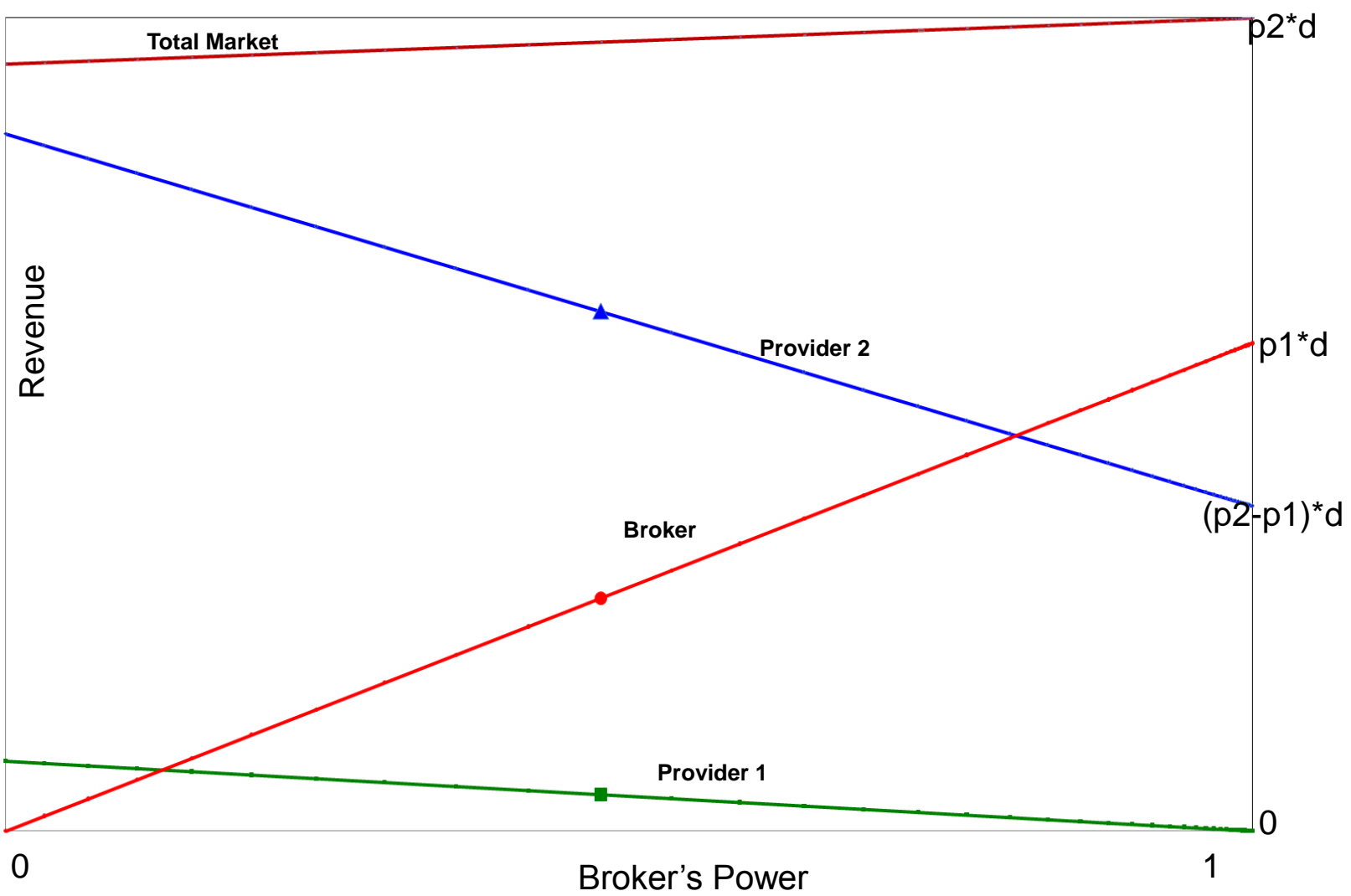

Figure 5 Large capacities with thresholds, $p_{1}=5, p_{2}=3, d=11$

is not competitive, the revenue split does not change by introducing sale thresholds. However, in a competitive market, the revenue split changes in the presence of thresholds, as stated in the following theorem.

THEOREM 5. The introduction of thresholds has the following effects in a competitive market:

- The profits of the larger provider increase or stay the same,

- The profits of the smaller provider decrease or stay the same,

- The profits of the broker can either increase, decrease or stay the same.

Recall that our definition of the larger provider is that with larger sellable capacity. It is interesting to see how the introduction of thresholds changes profits in a way that may not be consistent with intuition. Intuition suggests that the broker should fear thresholds because with them he may have to work for free. Our analysis suggests that it is the smaller provider the one who stands to lose the most by the introduction of thresholds. Comparing Figures 2 and 4 over the interval $\left(c_{1}, c_{1}+c_{2}\right)$ shows that the losses for the smaller provider can be very substantial in a non-loyal market and that the benefits go essentially to the broker. Notice also that the larger provider can introduce thresholds with the purpose of hurting the smaller provider even when he may not directly benefit. 
Although the broker can either win or lose, we have identified a situation when he always wins from the introduction of thresholds. This is when competition is so keen and the products are so similar that prices are equal. The intuition here is that the introduction of thresholds forces a fiercer competition through margins that end up benefiting the broker.

\subsection{Exogenous Commission Margins}

We have seen that the broker may benefit from the introduction of the thresholds, but in practice brokers adamantly resist the introduction of sales thresholds. How can this paradox be explained? Our best explanation is to consider the framework where thresholds are introduced in an environment where margins are fixed. This is in fact what has happened in the airline industry where historical margins that were paid on all sales are now paid only when sales exceed a threshold. Should the broker be anxious about the introduction of thresholds in this situation? If so, who benefits from the introduction of thresholds?

The assumption of zero partial commission margins remains without loss of generality, as stated in the following theorem.

THEOREM 6. We can assume, without loss of generality, that partial commission margins are zero, as there does not exist an equilibrium with positive partial commission margins with higher revenues for the providers.

We present the proof in the Appendix. An important insight that can be gleaned from the proof is that having a positive partial commission margin may help the competitor become the primary provider and since neither of the providers prefers to become the secondary provider, they both prefer to have zero commission margins below their thresholds.

However, buying and discarding unsold items may become necessary in equilibrium when margins are exogenous. Let $\delta_{i}$ denote the number of units of provider $i$ discarded by the broker, the broker's formulation is given by:

$$
\begin{array}{rlrl}
\pi_{B}\left(t_{i}\right)= & \max _{\left(s_{i}, k_{i}, \delta_{i}\right)} \sum_{i=1,2}\left[k_{i} m_{i}\left(s_{i}+\delta_{i}\right)-p_{i} \delta_{i}\right] & \\
& r_{i} \leq s_{i} \leq b_{i} & & \text { for } i=1,2 \\
& k_{i} t_{i} \leq s_{i}+\delta_{i} \leq c_{i} & & \text { for } i=1,2 \\
& s_{1}+s_{2} \leq d & \\
& k_{i} \in\{0,1\} & & \text { for } i=1,2 \\
& 0 \leq \delta_{i} & & \text { for } i=1,2 .
\end{array}
$$


Regarding the providers' formulation, the only decision variable is the threshold $t_{i}$, so the providers' formulation is given by:

$$
\begin{gathered}
\pi_{i}\left(t_{j}\right)=\max _{t_{i}}\left(p_{i}-k_{i} m_{i}\right) s_{i} \\
r_{i} \leq t_{i} \leq c_{i} \\
0 \leq m_{i} \leq p_{i}
\end{gathered}
$$

In monopoly, the provider can sell as much as $\min [c, p d /(p-m)]$ and earn revenue of $\min [(p-$ $m) c, p d]$. This implies the provider, conditioned on having enough capacity, can inflate the demand by a factor of $\frac{p}{p-m}$ by requiring a threshold higher than the market size. On the basis of this intuition, we use the following definitions to analyze the equilibrium.

Definition 3. Define

- We say that provider $i$ has ample capacity if $c_{i}>\frac{p_{i}}{p_{i}-m_{i}} r_{i}$, and has scarce capacity otherwise.

- $\hat{s}_{i}=\max \left[b_{i}, \frac{p_{i}}{p_{i}-m_{i}} r_{i}\right]$

- $\hat{\pi}_{i}=m_{i} \hat{s}_{i}-p_{i}\left(\hat{s}_{i}-b_{i}\right)$

Notice that as products become closer substitutes and the loyal demands decrease, it is more likely that providers capacity exceeds loyal demand, intensifying competition for the non-loyal portion of the demand. Also, notice also that $p_{i} /\left(p_{i}-m_{i}\right)$ decreases when $p_{i}$ increase or $m_{i}$ decreases. A lower $p_{i} /\left(p_{i}-m_{i}\right)$ implies a more intense competition since providers can inflate their demand less. Therefore, as the market price increases or full margins decrease, competition intensifies. We focus on the case that both providers have ample capacity: $c_{i}>\frac{p_{i}}{p_{i}-m_{i}} r_{i}$ for $i=1,2$, and call it a competitive market under exogenous commission margins. We label the provider with higher $\hat{\pi}_{i}$, provider 1 . We break the ties such that the first provider be the preferred one.

THEOREM 7. When commission margins are exogenous, there exists a pure strategy equilibrium such that $t_{1}^{*}=\min \left[c_{1}, \hat{s}_{1}+\frac{\hat{\pi}_{1}-\hat{\pi}_{2}}{p_{1}-m_{1}}\right]$ and $t_{2}^{*}=\frac{p_{2}}{p_{2}-m_{2}} r_{2}$ which results in revenue split:

$$
\pi_{1}^{*}=\min \left[\left(p_{1}-m_{1}\right) c_{1}, p_{1} b_{1}-\hat{\pi}_{2}\right] \quad \pi_{2}^{*}=p r_{2} \quad \pi_{B}^{*}=\max \left[m_{1} c_{1}-p_{1}\left(c_{1}-b_{1}\right), \hat{\pi}_{2}\right]
$$

Note that each provider sets a threshold at least as high as $p_{i} r_{i} /\left(p_{i}-m_{i}\right)$ and the broker reaches only provider 1 's threshold. Theorem 7 states that the provider with higher $\hat{\pi}$ becomes primary, so larger full commission margin, higher available capacity, and higher size of market loyal share are all criteria for getting prioritized. We can interpret $\hat{\pi}_{i}$ as the maximum total commission fee that provider $i$ is ready to pay the broker. As Theorem 7 confirms, the broker assigns the inflated residual demand to the secondary provider. It also confirms that when providers have ample capacity, discarding is inevitable when $c_{1}>d_{0}+d_{1}$, unless $p_{1} r_{1} /\left(p_{1}-m_{1}\right)<d_{0}+d_{1}$ and $\hat{\pi}_{1}=\hat{\pi}_{2}$. 
It follows from the proof of Theorem 7 that while an equilibrium always exists, it is not necessarily unique. We observe that in certain cases, there are equilibria with and without free ride that generate the same revenues for providers and the broker. In other words, providers can attain as much revenue from more sales, as they can from free ride, assuming selling is advantageous for the broker. We mentioned one equilibrium in the the theorem; the set of all equilibria can be found in the proofs.

To be able to investigate the effect of the thresholds, we study the case without thresholds and fixed commission margins. If both providers do not require thresholds, the provider with higher full commission margin is the primary one, and revenue splits as $\pi_{1}^{o}=\left(p_{1}-m_{1}\right) b_{1}, \pi_{2}^{o}=\left(p_{2}-m_{2}\right) r_{2}$, and $\pi_{B}^{o}=m_{1} b_{1}+m_{2} r_{2}$. It is an intuitive result since given the exogenous margins, the broker selects the provider with the higher margin and allocates as much demand as possible to it and gives the residual demand to the secondary provider. In other words, the broker's only criterion to prioritize a provider is a higher margin; market loyal share and capacity are not criteria. As expected, without thresholds, discarding does not happen.

The following theorem summarizes the strategic effects of the thresholds and how the market revenue is split between the broker and the providers.

THEOREM 8. In a market with exogenous commission margins:

- Threshold are effective unless $c_{i} \leq d_{0}+d_{i}$ for $i=1,2$ and $c_{1}+c_{2} \leq d$,

- When the thresholds are effective, the profits of the broker decrease,

- When the thresholds are effective, the profits of the provider with the lower margin increases unless her capacity is less than her loyal demand,

- When the thresholds are effective, the profits of the provider with the higher margin can either increase, decrease or stay the same.

As the theorem shows when the thresholds are effective, the provider with the smaller commission margin always wins and the broker always loses, as a result of threshold introduction. The fate of provider with higher commission margin depends on capacities and market structure, and is detailed in the Appendix.

Notice that these results hold if thresholds are effective. Fixing the size of the market, as the broker becomes more powerful and therefore $d_{0}$ gets larger, the thresholds start to become effective. This insight can explain the birth of sales thresholds in airline industry: as some travel agents became very powerful, some service providers started experimenting with positive thresholds to trigger commissions.

The introduction of thresholds reduces the flexibility of the broker to allocate non-loyal demand among the providers. Without thresholds, if commission margins are equal, then any allocation 
of non-loyal demand among the providers results in the same profit for the broker. However with thresholds, even if the total commission fees are equal, the broker is likely to use an extreme point solution to allocate non-loyal demand.

As explained before, the case of fixed margins and thresholds can be justified in the short-term situation where full margins are fixed and the providers are just manipulating the sales thresholds. With thresholds, the provider that is ready to pay a higher total commission gets prioritized. The total commission depends on the available quantity and the market loyalty as well as the commission margin. Our analysis shows that in a competitive market under exogenous margins, the provider with lower full commission margin wins by introducing thresholds. This result shows that at least one of the providers has an incentive to introduce thresholds. The other provider either wins or loses; and the broker always loses. Moreover, we show that with exogenous commission margins, there are cases in which discarding is inevitable.

This result is in sharp contrast to the case of endogenous markets. The paradox can be stated as follows: in the short term and with exogenous margins, the smaller provider wins at the expense of the broker by introducing thresholds. This justifies the broker's fears. In the long-term and with endogenous margins, the larger provider wins, the smaller loses and the broker's fate depends on the market structure.

\section{Conclusions and Further Research}

We have analyzed a setting in which two providers sell their partially substitutable products through a broker and each provider can decide on her commission margin schedule. This analysis enabled us to consider horizontal competition between providers as well as vertical competition between providers and the broker.

We used Game Theory to analyze the interactions and presented the results in the Nash framework, where providers make their decisions simultaneously, without knowledge of the other provider's decision. A set of actions is a Nash equilibrium, N.E., if each provider's action is the best response to the other one. While we present the analyses in the Nash framework, the main results are also valid in the Stackelberg framework, where the follower chooses her actions after observing the leader's actions. In other words, revenue distribution in the Stackelberg equilibrium. S.E., is independent of the leadership and is identical to the revenue distribution in Nash equilibrium. To be more precise, while the strategies, margins and thresholds, can be different in N.E. and S.E, the players' revenues are the same. It is evidence that the revenue shares of the providers and the broker are quite robust and the fact that who is leader does not matter and the results of S.E. and N.E. are identical. The only exception is when the margins are endogenous and the thresholds do not exist. Regardless, our analysis about the effects of requiring thresholds remains valid. For a more detailed analysis, refer to Talebian (2010). 
We have shown that without loss of generality we can limit the commission margin schedules to a simple schedule, where each provider requires a sales threshold to trigger full commission margins for all units sold. In this setting, we investigated the effect of requiring a sales threshold on how the revenue splits between providers and the broker. In a competitive market, thresholds affect how revenue is split between the players. We showed that in equilibrium the larger provider offers a discount schedule with a higher threshold and a smaller commission margin. As a result of the thresholds, the larger provider, the provider with larger capacity and/or loyal demand, wins at the expense of the smaller provider. Our results show that the broker may benefit or disbenefit from introducing the thresholds, depending on marker structure. While the loss cases may be more intuitive, the gains can be explained since when providers compete both on the thresholds and commission margins there are two competition levers which further increases the level of competition. The comparative statics show that the changes in profit as a result of demand changes are capped by prices, and therefore the equilibrium is smooth to changes in demand.

Our analysis shows that when full margins are exogenous, at least one provider has an incentive to require minimum sales volumes. However, the apparent gains are just a mirage, since this equilibrium is not sustainable as providers have then an incentive to change their commission margins. Comparing equilibria with and without thresholds under endogenous commission margin shows that imposing thresholds is not Pareto optimal and one of the providers loses.

In both settings, as was expected, the equilibrium depends on comparative size of the market to capacities and the market price. As observed, thresholds are also effective in a competitive market, where supply is large compared to demand. This implies that if the market is too large compared to the available capacities, then there is no competition between providers and thresholds are not effective.

While the providers' revenue may decrease or increase as the broker becomes more powerful, in all cases, the broker's revenue is increasing as his power increases. While it confirms our intuition, it is not trivial. Notice that with fixed providers' actions, more power implies more freedom in assigning the sale, a larger feasible region for his optimization problem. However, as the broker's power increases, the providers also change their policy. Another observation corresponds to the broker's revenue change as the demand increases. Market growth is not necessarily beneficial for the broker, since more demand results in less competition and lower commission margins.

We consider the special case where the providers' capacities are large such that they are not limiting factors. This case closely represents service providers like insurance companies, where capacity is virtually infinite. Notice that in this case the capacity is not binding; $c_{i}>d_{0}+d_{i}$. Our study shows that as the broker's power increases and demand becomes non-loyal the broker's 
revenue increases. This can help explaining the very large commission margins of brokers in the insurance industry when they sign in new customers.

As discussed, contracts without thresholds are unstable and in equilibrium the providers require minimum sales volumes, which means that competition can justify the use of sophisticated contracts. In contrast, the main reason to use sophisticated contracts in the operations management literature is to increase coordination, e.g., avoid double marginalization in pricing and news-vendor settings. We think our result is quite different from the traditional view since we introduced a setting that the competition results in a sophisticated contract. We pay special attention to the specific case of equal prices. In this case, the total revenue of a competitive market is fixed at $p d$, and does not depend on thresholds. It only depends on the capacities and the sizes of the market, and there is no revenue losses because of decentralized decision making. However, still our study confirms that competition, without any notion of coordination, justifies requiring thresholds.

We also believe that this work can shed light into the effects of business contracts in a competitive setting. We think our approach to competition may question some of the core insights of traditional models; in particular, the emphasis on negotiation on allocating coordination gains. Negotiation plays an important role in most contracts without competition because usually there is not a unique way to distribute the additional profits gained from the coordination between the chain members. However, in a competitive setting, the gains are divided specifically because of a more limited number of equilibria and this lowers the effect of negotiation on the outcome of the game.

In our work, we presented competition, in addition to coordination, as a justification for the sophisticated contracts. It introduces a new potential area of research, which explores settings in which both coordination and competition exist. We discuss a few avenues of this area here. First, an avenue of research can be suggested by making the model more realistic by considering the providers' marginal costs of production, which can be heterogeneous and production volume dependent. Similarly, one can consider the broker's distribution costs. Another setting, in which the chain coordination can be investigated, is to study a game where the broker has the pricing power and/or should decide the level of his costly sales effort. This extensions make the model more realistic and also add significant complexity to the basic model.

In the long term, some fixed parameters also can change and become decision variables. In a more general setting, not only the providers can decide about commission margin schedules, but about their capacities, loyal shares, and prices too. We can think of a two stage game, in which providers decide about these parameters in the first stage and then decide about their commission margin schedule.

Also, a stochastic model, where the demand has a probabilistic distribution, can be considered. We showed that the results change smoothly in regard to demand volume; however, the equilibrium 
with random demand can be quite different from that with a deterministic model. Notice that in a stochastic setting, unlike a deterministic one, contracts regarding commission on order differ from the ones about commission on sale.

Finally, the identification of threshold paradox, the fact that in some settings the broker benefits from introduction of thresholds but still resists thresholds introduction, has the potential to attract more research attention. One possibility is looking more closely at practical contexts in which the broker's resistance exist and check whether the theory predicts the broker's loss or win. We looked at a specific setting where products prices are equal and showed that the broker wins.

To explain the threshold paradox, we studied short term effects, when commission margins are fixed. This justification relies on assuming shallow analytic capabilities of some brokers and not being able to study more complicated effects. However, one can explore other avenues to justify the paradox. One possible explanation may be based on renegotiation element in contracts; the fact that if the broker and one of the providers find that they are both mutually better off by renegotiating the current contract to a different contract, they will credibly do so. The fact that our results are also valid in the Stackelberg framework may give some confidence that the current equilibria are negotiation-proof.

\section{References}

Alamdari, F. 2002. Regional development in airlines and travel agents relationship. Journal of Air Transport Management 8(5).

Aviv, Y. 2001. The effect of collaborative forecasting on supply chain performance. Management Science 47(10) $1326-1343$.

Boyaci, Tamer, Guillermo Gallego. 2004. Supply chain coordination in a market with customer service competition. Journal of Air Transport Management 13(1) 3-22.

Cachon, Gérald P. 2003. Supply chain coordination with contracts. S. Graves, T. de Kok, eds., Handbook in OR/MS: Supply Chain Management. North-Holland.

Cachon, Gérald P., A. Gurhan Kok. 2010. Competing manufacturers in a retail supply chain: On contractual form and coordination. Management Science 56(3) 571-589.

Cournot, Antoine Augustin. 1838. Recherches sur les principes mathmatiques de la thorie des richesses. Paris.

Fry, J. M., R. Kapuscinski, T. L. Olsen. 2001. Coordinating production and delivery under a (z, z)-type vendor-managed inventory contract. Manufacturing Service Oper. Management 151-173.

Gallego, G., C. Stefanescu. 2009. Upgrades, upsells and pricing in revenue management. Working Paper, IEOR Department, Columbia University, NY, http://ssrn.com/abstract=1334341 . 
Hax, A. C, D. Candea. 1984. Productiona and Inventory Management. Prentice-Hall, Inc., Englewood Cllffs, NJ.

Heese, H. S. 2008. Pitch it now or pitch it later? extended warranty sales strategies and the impact on manufacturer warranties. Working paper .

Jeuland, Abel P., Steven M. Shugan. 1983. Managing channel profits. Marketing Science 2(3) 239-272.

Kinter, Earl W. 1970. A Robinson-Patman Primer. Macmillan, New York.

Klein, B., R. G. Crawford, A. A. Alchian. 1978. Vertical integration, appropriable rents, and the competitive contracting process. The Journal of Law and Economics 21 297-326.

Koabiyikoglu, A., I. Popescu, C. Stefanescu. 2010. Pricing and revenue management with stochastic demand: Coordinated versus hierarchical approaches. working paper. .

Lariviere, M. A. 1999. Supply chain contracting and coordination with stochastic demand. R. Ganeshan S. Tayur, M. Magazine, ed., Quantitative Models of Supply Chain Management. Kluwer Academic Publishers, Boston, MA, 233-268.

Mahajan, S., G. J. van Ryzin. 2001. Inventory competition under dynamic consumer choice. Operetions Research 49(5) 646-657.

Spengler, J. J. 1950. Vertical integration and anti-trust policy. The Journal of Political Economy 58(4) $347-352$.

Talebian, Masoud. 2010. Ph.D. Thesis: Essays on Pricing and Contract Theory. Columbia University, New York, NY.

Talluri, K.T., G. J. van Ryzin. 2004. The Theory and Practice of Revenue Management. Kluwer Academic Publishers.

Tirole, J. 1988. The Theory of Industrial Organization. MIT Press, Boston, MA.

Weng, Z. K. 1995. Channel coordination and quantity discount. Management Science 41(9) 1509-1522.

\section{Appendix}

We discuss the proofs in a different order from their appearance in the paper to get advantage of the mathematical relation between them. First we start with the proofs about exogenous margins and then present the proofs about endogenous margins.

We define $0 \leq w_{i} \leq m_{i}$ as the partial commission margin that provider $i$ pays the broker in case that he does not reach the threshold, and $\delta_{i}$ as the number of units of provider $i$ discarded by the broker. Incorporating partial commission margins and discarding, the complete formulations for the broker and the providers are given by: 


$$
\begin{aligned}
& \pi_{B}\left(w_{1}, w_{2}, t_{1}, t_{2}, m_{1}, m_{2}\right)=\max _{\left(s_{i}, k_{i}, \delta_{i}\right)} \sum_{i=1,2}\left[\left(1-k_{i}\right) w_{i}\left(s_{i}+\delta_{i}\right)+k_{i} m_{i}\left(s_{i}+\delta_{i}\right)-p_{i} \delta_{i}\right] \\
& r_{i} \leq s_{i} \leq b_{i} \\
& k_{i} t_{i} \leq s_{i}+\delta_{i} \leq c_{i} \\
& s_{1}+s_{2} \leq d \\
& k_{i} \in\{0,1\} \\
& 0 \leq \delta_{i} \\
& \text { for } i=1,2 \\
& \text { for } i=1,2 \\
& \text { for } i=1,2 \\
& \text { for } i=1,2 \text {. } \\
& \pi_{i}\left(w_{j}, t_{j}, m_{j}\right)=\max _{\left(w_{i}, t_{i}, m_{i}\right)}\left[p_{i}-\left(1-k_{i}\right) w_{i}-k_{i} m_{i}\right]\left(s_{i}+\delta_{i}\right) \\
& r_{i} \leq t_{i} \leq c_{i} \\
& 0 \leq w_{i} \leq m_{i} \leq p_{i}
\end{aligned}
$$

With exogenous full commission margins, $m_{1}$ and $m_{2}$ will be fixed and no longer are providers' decision variables.

We use $a_{i}=s_{i}+\delta_{i}$ in the proofs to make the notation easier to follow. Notice that $a_{i}$ equals all units which provider $i$ delivers through the broker, whether sold or discarded.

\section{A. Exogenous Commission Margin}

We first study the case where both margins $0 \leq w \leq m$ are fixed and providers decide only the thresholds. The next three propositions investigate this one dimensional game. We start with the following proposition, where a single provider selects its sales threshold.

PROPOSITION 1. Under the assumption that commission margins are exogenous, the single provider's best sales threshold is $t^{*}=\min \left[c, \frac{p-w}{p-m} d\right]$ which results in sales:

$$
s^{*}=b, \delta^{*}=t^{*}-b
$$

and revenue split:

$$
\pi^{*}=\min [(p-m) c,(p-w) d] \quad \pi_{B}^{*}=\max [m c-p(c-d), w d] .
$$

Proof Recall that $b=\min (c, d)$ where $d$ is the total demand. Since $w \leq m$, the form of $t^{*}$ implies that $\delta^{*}=t^{*}-b \geq 0$. The broker prefers to reach the threshold whenever $m t-p(t-d)^{+} \geq w \min [t, d]$, by setting $a=t$. Since the provider earns $(p-m) a$, it is optimal for the provider to choose the largest $t \leq c$ which satisfies the above inequality. Therefore, the provider chooses $t^{*}=\min \left[c, \frac{p-w}{p-c} d\right]$, and earns $(p-m) t^{*}=$ $\min [(p-m) c,(p-w) d]$, which leaves the broker with the remaining revenue of $p d-\min [(p-m) c,(p-w) d]=$ $\max [p d-(p-m) c, w d]$.

If $c>\frac{p-w}{p-m} d$, then the provider also can set higher thresholds $t^{*} \in\left(\frac{p-w}{p-m} d, c\right]$. In this case, the broker does not reach the threshold and sets $a^{*}=d$, so the provider gets a free ride. However, this does not change the revenue split.

If $c<d$, then setting a threshold is not important for the provider and $t^{*} \in[0, c]$. 
DEFINITION 4. We say that provider $i$ has ample capacity if $c_{i}>\frac{p_{i}-w_{i}}{p_{i}-m_{i}} r_{i}$, and has scarce capacity otherwise.

Proposition 2. Suppose that provider 1 has scarce capacity. Then, there exists a pure strategy equilibrium such that $t_{1}^{*}=c_{1}$ and $t_{2}^{*}=\min \left[c_{2}, \frac{p_{2}-w_{2}}{p_{2}-m_{2}} r_{2}\right]$ which results in sales:

$$
s_{1}^{*}=b_{1} \quad \delta_{1}^{*}=c_{1}-b_{1} \quad s_{2}^{*}=r_{2} \quad \delta_{1}^{*}=\min \left[c_{2}, \frac{p_{2}-w_{2}}{p_{2}-m_{2}} r_{2}\right]-r_{2}
$$

and revenue split:

$$
\begin{gathered}
\pi_{1}^{*}=\left(p_{1}-m_{1}\right) c_{1} \quad \pi_{2}^{*}=\min \left[\left(p_{2}-m_{2}\right) c_{2},\left(p_{2}-w_{2}\right) r_{2}\right] \\
\pi_{B}^{*}=m_{1} c_{1}-p_{1}\left(c_{1}-b_{1}\right)+\max \left[m_{2} c_{2}-p_{2}\left(c_{2}-r_{2}\right), w_{2} r_{2}\right] .
\end{gathered}
$$

Proof Notice that $c_{1} \leq \frac{p_{1}-w_{1}}{p_{1}-m_{1}} r_{1} \Rightarrow w_{1} r_{1} \leq m_{1} c_{1}-p_{1}\left(c_{1}-r_{1}\right)$, and hence the broker is always better off reaching the first provider's threshold. Since $\pi_{1}=\left(p_{1}-m_{1}\right) s_{1}=\left(p_{1}-m_{1}\right) t_{1}$, provider 1 is setting $t_{1}$ as high as possible, i.e., to $t_{1}^{*}=c_{1}$. This means the broker can maximize his revenue by selling $a_{1}^{*}=t_{1}^{*}=c_{1}$. After selling the first provider's capacity, the second provider is facing a monopolistic market of size $r_{2}$, so based on Proposition $1 t_{2}^{*}=\min \left[c_{2}, \frac{p_{2}-w_{2}}{p_{2}-m_{2}} r_{2}\right]$.

LEMMA 1. Consider a case where both providers have ample capacity with exogenous commission margins. Then, w.l.o.g., we can limit the strategy of provider $i$ to $t_{i}>\frac{p_{i}-w_{i}}{p_{i}-m_{i}} r_{i}$. In addition, the broker reaches at most one of the providers' thresholds in equilibrium and if he reaches the threshold of provider $i$, then $s_{i}+\delta_{i} \geq b_{i}$.

\section{Proof W.l.o.g., $t_{i}>\frac{p_{i}-w_{i}}{p_{i}-m_{i}} r_{i}$ :}

Since the providers have ample capacity, provider $i$ can guarantee a minimum revenue of $\left(p_{i}-w_{i}\right) r_{i}$ by setting $t_{i}>\frac{p_{i}-w_{i}}{p_{i}-m_{i}} r_{i}$. If provider $i$ chooses $t_{i} \leq \frac{p_{i}-w_{i}}{p_{i}-m_{i}} r_{i}$ and the broker reaches her threshold by setting $a_{i}=t_{i}$, her revenue is given by $\left(p_{i}-m_{i}\right) t_{i}$ which is less than or equal to the minimum guaranteed, $\left(p_{i}-w_{i}\right) r_{i}$ and this implies that $t_{i}>\frac{p_{i}-w_{i}}{p_{i}-m_{i}} r_{i}$.

The broker never reaches both providers' thresholds in equilibrium. Label the provider with higher price $p_{1}$. Notice that:

- Reaching both thresholds, $\left(t_{1} \leq a_{1}, t_{2} \leq a_{2}\right)$ :

$$
\begin{aligned}
\pi_{B} & =p_{1} s_{1}-\left(p_{1}-m_{1}\right) a_{1}+p_{2} s_{2}-\left(p_{2}-m_{2}\right) a_{2} \\
& <p_{1} s_{1}-\left(p_{1}-m_{1}\right) a_{1}+p_{2} s_{2}-\left(p_{2}-w_{2}\right) r_{2} \\
& =p_{1} s_{1}-\left(p_{1}-m_{1}\right) a_{1}+p_{2}\left(s_{2}-r_{2}\right)+w_{2} r_{2} \\
& =p_{1} b_{1}-\left(p_{1}-m_{1}\right) a_{1}+w_{2} r_{2}
\end{aligned}
$$

- Reaching the threshold of provider $1,\left(t_{1} \leq a_{1}, a_{2}<t_{2}\right)$ :

$\pi_{B} \geq p_{1} b_{1}-\left(p_{1}-m_{1}\right)\left(\max \left[t_{1}, b_{1}\right]\right)+w_{2} r_{2}$ 
To prove the claim by contradiction, assume that reaching both thresholds is better than reaching only the threshold of provider 1 in equilibrium, then:

$$
\begin{array}{ll} 
& p_{1} b_{1}-\left(p_{1}-m_{1}\right) a_{1}+w_{2} r_{2}>p_{1} b_{1}-\left(p_{1}-m_{1}\right)\left(\max \left[t_{1}, b_{1}\right]\right)+w_{2} r_{2} \\
\Rightarrow & a_{1}<\max \left[t_{1}, b_{1}\right] \\
\Rightarrow \quad & a_{1}<b_{1} \quad\left(\text { since } t_{1} \leq a_{1}\right)
\end{array}
$$

In this case, provider 1 has an incentive to increase her threshold to more than her current sales and still be sure that the broker reaches her threshold. It shows that the current point was not an equilibrium and results in a contradiction.

W.l.o.g., we can assume that in equilibrium $a_{i} \geq t_{i} \Rightarrow a_{i} \geq b_{i}$ :

Assume for a contradiction that $t_{i} \leq a_{i}<b_{i}$. Since provider $i$ has responded optimally, if it increases $t_{i}$ up to $a_{i}+\epsilon$, the broker does not reach her threshold. One of these cases must happen:

- The broker reaches none of the thresholds, $m_{i} a_{i}+w_{j}\left(d-a_{i}\right)=\max \left[w_{i} r_{i}+w_{j} b_{j}, w_{i} b_{i}+w_{j} r_{j}\right]=w_{i} r_{i}+w_{j} b_{j}$ : Provider $j$ can put $t_{j}^{\prime}=\frac{p_{j}-w_{j}}{p_{j}-m_{j}} b_{j}$ and be sure that the broker reaches her threshold, because $m_{j} l_{j}^{\prime}-p_{j}\left(t_{j}^{\prime}-\right.$ $\left.b_{j}\right)+w_{i} r_{i}=w_{j} b_{j}+w_{i} r_{i}=m_{i} a_{i}+w_{j}\left(d-a_{i}\right)$. This means the current point is not an equilibrium, noting $\left(p_{j}-w_{j}\right) b_{j}>\left(p_{j}-w_{j}\right)\left(d-a_{i}\right)$.

- The broker reaches the threshold of provider $j: m_{i} a_{i}+w_{j}\left(d-a_{i}\right)=w_{i} r_{i}+m_{j} \max \left[t_{j}, b_{j}\right]-p_{j}\left(t_{j}-b_{j}\right)^{+}$ It means the broker is indifferent between satisfying $t_{i}$ by selling $a_{i}$, or satisfying $t_{j}$ by selling $\max \left[t_{j}, b_{j}\right]$ and both are equilibrium.

DEFINITION 5. Let $\hat{s}_{i}=\max \left[b_{i}, \frac{p_{i}-w_{i}}{p_{i}-m_{i}} r_{i}\right]$ and $\hat{\pi}_{i}=m_{i} \hat{s}_{i}-p_{i}\left(\hat{s}_{i}-b_{i}\right)+w_{j} r_{j}$.

$\hat{\pi}_{i}$ can be thought of as the revenue cap for the broker, conditioned on reaching the threshold of provider $i$. Proposition 3 studies the duopoly case where both providers have ample capacity, based on the results on Lemma 1 and Definition 5. We label the provider with higher $\hat{\pi}_{i}$, provider 1.

Proposition 3. Consider the case where both providers have ample capacity with exogenous commission margins. Then, there exists a pure strategy equilibrium such that $t_{1}^{*}=\min \left[c_{1}, \hat{s}_{1}+\frac{\hat{\pi}_{1}-\max \left[\hat{\pi}_{2}-w_{2} r_{2}, w_{1} b_{1}\right]}{p_{1}-m_{1}}\right]$ and $t_{2}^{*}=\frac{p_{2}-w_{2}}{p_{2}-m_{2}} r_{2}$ which results in sales:

$$
s_{1}^{*}=b_{1} \quad \delta_{1}^{*}=t_{1}^{*}-b_{1} \quad s_{2}^{*}=r_{2} \quad \delta_{2}^{*}=0
$$

and revenue split:

$$
\begin{gathered}
\pi_{1}^{*}=\min \left[\left(p_{1}-m_{1}\right) c_{1}, p_{1} b_{1}-\max \left[\hat{\pi}_{2}-w_{2}\left(d-b_{1}\right), w_{1} b_{1}\right]\right] \quad \pi_{2}^{*}=\left(p_{2}-w_{2}\right) r_{2} \\
\pi_{B}^{*}=\max \left[m_{1} c_{1}-p_{1}\left(c_{1}-b_{1}\right), \hat{\pi}_{2}-w_{2} r_{2}, w_{1} b_{1}\right]+w_{2} r_{2}
\end{gathered}
$$

Proof Let's define $\pi_{R i}$ as the broker's revenue, conditioned on reaching the threshold of provider $i$. Based on Lemma 1, the broker does not reach both thresholds in equilibrium, the broker chooses one of the four actions after observing the providers' thresholds:

$$
\pi_{B}=\max \left[\pi_{R 1}, \pi_{R 2}, w_{1} b_{1}+w_{2} r_{2}, w_{1} r_{1}+w_{2} b_{2}\right]
$$


The first two alternatives correspond to reaching the threshold of the first or the second provider. The last two alternatives correspond to not reaching any of the thresholds and giving priority to the first or the second provider. On the basis of Lemma 1, we know that in equilibrium, $a_{i}<t_{i}$ or $a_{i} \geq \hat{s}_{i}$. We now show that $\pi_{R i} \leq \hat{\pi}_{i}$ for $i=1,2$. Since if $a_{i} \geq t_{i}$ then $a_{i} \geq \hat{s}_{i}$, the broker's revenue from provider $i$ is capped by $m_{i} \hat{s}_{i}-p_{i}\left(\hat{s}_{i}-b_{i}\right)=m_{i} b_{i}-\left(p_{i}-m_{i}\right)\left(\hat{s}_{i}-b_{i}\right)$, and his revenue from provider $j$ is capped by $w_{j} r_{j}$. Therefore, his total revenue after reaching $t_{i}$ is capped by $\hat{\pi}_{i}$.

Now, in response to provider $i$, provider $j$ should decide about $t_{j}$ which determines $\pi_{R j}$. Provider $j$ has two options: fight back to become the primary provider, or allow provider $i$ to become primary, which correspond to:

1. $\pi_{R j} \geq \max \left[\pi_{R i}, w_{1} b_{1}+w_{2} r_{2}, w_{1} r_{1}+w_{2} b_{2}\right]: \pi_{i}=\left(p_{i}-w_{i}\right) r_{i}, \pi_{j}=p_{j} b_{j}-\pi_{R j}$

2. $\pi_{R j}<\max \left[\pi_{R i}, w_{1} b_{1}+w_{2} r_{2}, w_{1} r_{1}+w_{2} b_{2}\right]: \pi_{i}=p_{i} b_{i}-\pi_{R i}, \pi_{j}=\left(p_{j}-w_{j}\right) r_{j}$

It is easy to show that the strategy 1 is always more beneficial for provider $j$, and hence it always chooses strategy 1 if it can. This implies that providers $i$ and $j$ put $\pi_{R i}$ and $\pi_{R j}$ as high as it is necessary to beat the other provider. Therefore, the result of the game will depend on the relation between $\hat{\pi}_{i}$ and $\hat{\pi}_{j}$. Since we have labeled provider 1 to have the highest revenue cap, $\hat{\pi}_{1} \geq \hat{\pi}_{2}$, we have:

$$
\begin{aligned}
\pi_{R 1} & \geq \max \left[\pi_{R 2}, w_{1} b_{1}+w_{2} r_{2}, w_{1} r_{1}+w_{2} b_{2}\right] \\
\Rightarrow \pi_{R 1} & \geq \max \left[\hat{\pi}_{2}, w_{1} b_{1}+w_{2} r_{2}\right] \quad\left(w_{1} r_{1}+w_{2} b_{2} \leq \hat{\pi}_{2}\right) \\
\Rightarrow p_{1} b_{1}-\left(p_{1}-m_{1}\right) t_{1}+w_{2} r_{2} & \geq \max \left[\hat{\pi}_{2}, w_{1} b_{1}+w_{2} r_{2}\right] \\
\Rightarrow t_{1} & \leq \frac{p_{1} b_{1}-\max \left[\hat{\pi}_{2}-w_{2} r_{2}, w_{1} b_{1}\right]}{p_{1}-m_{1}} \\
\Rightarrow t_{1} & =\min \left[c_{1}, \frac{p_{1} b_{1}-\max \left[\hat{\pi}_{2}-w_{2} r_{2}, w_{1} b_{1}\right]}{p_{1}-m_{1}}\right]
\end{aligned}
$$

After selling the primary provider's capacity, the second provider is facing a monopolistic market of size $r_{2}$, so by Proposition $1, t_{2}^{*}=\min \left[c_{2}, \frac{p_{2}-w_{2}}{p_{2}-m_{2}} r_{2}\right]$

\section{A.1. Theorem 6}

Theorem 6 studies the case where only full commission margins, $m_{1}$ and $m_{2}$, are fixed and providers can decide $t$ and $w$. In this case, we show that without loss of generality the providers can limit the analysis to $w=0$. More precisely, we show that for any equilibrium with positive $w$ there is one with $w=0$. We need the following lemma before proving Theorem 6 . We use $t_{i}\left(w_{i}, w_{j}\right)$ to denote the optimal threshold for given partial commission margins, which can be found based on Propositions 2 and 3.

LEMMA 2. Under the assumption that commission margins are exogenous, we have:

$$
\begin{gathered}
\pi_{1}\left(0, w_{2}, t_{1}\left(0, w_{2}\right), t_{2}\left(0, w_{2}\right)\right) \geq \pi_{1}\left(w_{1}, w_{2}, t_{1}\left(w_{1}, w_{2}\right), t_{2}\left(w_{1}, w_{2}\right)\right) \\
\pi_{1}\left(w_{1}, w_{2}, t_{1}, t_{2}\left(w_{1}, w_{2}\right)\right) \geq \pi_{1}\left(w_{1}, w_{2}, t_{1}, t_{2}\left(0, w_{2}\right)\right)
\end{gathered}
$$


Proof The first inequality can be proved by noticing that if provider 1 does not have ample capacity with $w_{1}>0$, it would not have ample capacity with $w_{1}=0$. Based on Definition $5, \hat{\pi}_{i}$ is a function of $\left(w_{i}, w_{j}\right)$. Since $\hat{\pi}_{1}\left(w_{1}, w_{2}\right) \leq \hat{\pi}_{1}\left(0, w_{2}\right)$ and $\hat{\pi}_{2}\left(w_{1}, w_{2}\right) \geq \hat{\pi}_{2}\left(0, w_{2}\right)$, if the first provider gets priority with $\left(w_{1}, w_{2}\right)$, it also gets priority with $\left(0, w_{2}\right)$. On the basis of these observations, by considering all different cases and some algebra, it can be shown that the lemma is true in each case. For a detailed table, refer to Talebian (2010)

The proof of the second inequality is similar to the first one, noting that $a_{2}\left(w_{1}, w_{2}\right) \leq a_{2}\left(0, w_{2}\right)$ and $t_{2}\left(w_{1}, w_{2}\right) \leq t_{2}\left(0, w_{2}\right)$.

Proof of Theorem 6 Based on the Proposition 3, we know $t_{1}\left(w_{1}, w_{2}\right)$ and $t_{2}\left(w_{1}, w_{2}\right)$ are Nash equilibrium strategies, when $w$ 's are fixed. Now, we are interested to find Nash equilibria when $\left(w_{i}, t_{i}\right)$ are chosen simultaneously. First note that any Nash equilibrium for the two dimensional game is in the format $\left(w_{1}, w_{2}, t_{1}\left(w_{1}, w_{2}\right), t_{2}\left(w_{1}, w_{2}\right)\right)$. If $\left(w_{1}^{*}, w_{2}^{*}, t_{1}^{*}, t_{2}^{*}\right)$ is a Nash equilibrium for the two dimensional simultaneous game, then, $t_{i}^{*}$ 's are equilibrium strategies for a one dimensional game which $w_{i}$ 's are fixed at $w_{i}^{*}$ and players only choose $t_{i}$ 's. On the basis of this necessary condition, we can take advantage of Proposition 3 , as if $\left(w_{1}^{*}, w_{2}^{*}, t_{1}^{*}, t_{2}^{*}\right)$ is a Nash equilibrium, then $\left(t_{1}^{*}, t_{2}^{*}\right)$ is a Nash equilibrium if $w_{1}=w_{1}^{*}$ and $w_{2}=w_{2}^{*}$.

Now, to justify that we can limit our study to find a Nash equilibrium of the two dimensional game to the case when $w_{1}=w_{2}=0$, we show that:

- $\left(0,0, t_{1}(0,0), t_{2}(0,0)\right)$ is a Nash equilibrium.

$$
\begin{array}{rlrl}
\pi_{1}\left(0,0, t_{1}(0,0), t_{2}(0,0)\right) & & \pi_{1}\left(w_{1}, 0, t_{1}\left(w_{1}, 0\right), t_{2}\left(w_{1}, 0\right)\right) & \text { Lemma } 2 \\
\geq & \pi_{1}\left(w_{1}, 0, t_{1}, t_{2}\left(w_{1}, 0\right)\right) & t_{1}\left(w_{1}, 0\right) \text { is the best response } \\
\geq & \pi_{1}\left(w_{1}, 0, t_{1}, t_{2}(0,0)\right) & \text { Lemma } 2
\end{array}
$$

- In any other Nash equilibrium, the players do not earn more. Using the proposition above, it is enough to show that: $\pi_{i}\left(w_{1}, t_{1}\left(w_{1}, w_{2}\right), w_{2}, t_{2}\left(w_{1}, w_{2}\right)\right) \leq \pi_{i}\left(0, t_{1}(0,0), 0, t_{2}(0,0)\right)$ for $i=1,2$ and $\forall 0 \leq w_{i} \leq m_{i}$ The proof is similar to Lemma 2

- In any other Nash equilibrium, one player can deviate to zero without losing.

$$
\begin{array}{rrrr}
\pi_{1}\left(w_{1}, w_{2}, t_{1}\left(w_{1}, w_{2}\right), t_{2}\left(w_{1}, w_{2}\right)\right) & & \pi_{1}\left(0, w_{2}, t_{1}\left(0, w_{2}\right), t_{2}\left(0, w_{2}\right)\right) & \text { Lemma } 2 \\
& \leq & \pi_{1}\left(0, w_{2}, t_{1}\left(0, w_{2}\right), t_{2}\left(w_{1}, w_{2}\right)\right) & \text { Lemma } 2
\end{array}
$$

Notice that Theorem 6 investigates the two dimensional game, where the providers can change both the lower commission margins and the thresholds. Theorem 6 states that without loss of optimality, we can limit our study to cases with zero lower commission margins and this allows us to reduce the two dimensional strategy space $\left(w_{i}, t_{i}\right)$ to a one dimensional space $t_{i}$. On the basis of this fact, we can use Propositions 1,2 , and 3 in the special case that $w_{i}=0$, for $i=1,2$.

\section{A.2. Theorem 7}

Proof Deduced from Proposition 3 when $w_{i}=0$ for $i=1,2$.

The case without thresholds can also be deduced from Proposition 3 when $w_{i}=m_{i}$ for $i=1,2$. 


\section{A.3. Theorem 8}

Proof - If $c_{i} \leq d_{0}+d_{i}$ and $c_{1}+c_{2} \leq d$, there will not be any competition between providers and, $a_{i}^{*}=c_{i}$.

- The broker loss can be confirmed through the formulation. Note that any feasible solution for with threshold scenario is a solution or can be capped by a solution for without threshold scenario.

$$
\begin{array}{ll}
c_{2}<d_{2} \Rightarrow & a_{2}^{*}=c_{2}(\text { with and without threshold) } \\
d_{2}<c_{2} \Rightarrow & a_{2}^{*}=r_{2}(\text { without threshold }) \\
& a_{2}^{*} \geq \min \left[\frac{p_{2}}{p_{2}-m_{2}} r_{2}, c_{2}\right] \text { (with threshold) }
\end{array}
$$

- We have:

without threshold: $a_{1}^{*}=b_{1}$

with threshold: $a_{1}^{*}=$

- Both providers have ample capacity:

$* 1$ is primary: $\min \left[c_{1}, \hat{s}_{1}+\frac{\hat{\pi}_{1}-\hat{\pi}_{2}}{p_{1}-m_{1}}\right]$

- $d_{0}+d_{1}<c_{1}:>b_{1}$ unless $\hat{\pi}_{1}=\hat{\pi}_{2}$ and $\frac{p_{1}}{p_{1}-m_{1}} r_{1}<d_{0}+d_{1}$

- $d_{0}+d_{1}>c_{1}: b_{1}$

$* 1$ is secondary: In this case, it needs to be $\frac{p_{1}}{p_{1}-m_{1}} r_{1}<d_{0}+d_{1}$, so the provider loses.

- At least one of the providers do not have ample capacity:

$* d_{0}+d_{1}<c_{1}:=\min \left[c_{1}, \frac{p_{1}}{p_{1}-m_{1}} r_{1}\right]>b_{1}$ if $d_{0}+d_{1}<\frac{p_{1}}{p_{1}-m_{1}} r_{1}$. Otherwise, it is lower.

$* c_{1} \leq d_{0}+d_{1}$ : If $c_{1} \leq \frac{p_{1}}{p_{1}-m_{1}} r_{1}$ then $c_{1}=b_{1}$. If $c_{1}>\frac{p_{1}}{p_{1}-m_{1}} r_{1}$ then $\frac{p_{1}}{p_{1}-m_{1}} r_{1}<b_{1}$

\section{B. Endogenous Commission Margins}

\section{B.1. Theorem 1}

Proof - Consider a non-negative and non-decreasing optimal commission margin function as $M(a)$ that results in a sale of $a^{*}$. Function $M$ can have jumps, and can be either left continuous or right continuous. We prove that for every $M(a)$ and $a^{*}$, there exists a commission margin schedule with one breakpoint that generates the same equilibrium. Consider a commission margin equal to 0 if $a<a^{*}$, and equal to $M\left(a^{*}\right)$ if $a \geq a^{*}$. We claim that the new commission margin, called $P(a)$ results in $a^{*}$. We prove the claim by contradiction.

To prove the claim, notice that $P(a) \leq M(a)$ with equality at $a=a^{*}$. If the new schedule results in sales $a \neq a^{*}$, then with the old schedule also point $a$ would be better than $a^{*}$. In other words, if there is another point that makes more profit for the provider than $a^{*}$ under $P$ then it will make at least as much profit under $M$.

Note that broker does not increase or decrease sales because he is facing the same or a smaller margin. Figure 6 shows the conversion.

- As before, we define $\pi_{R i}$ as the broker's revenue from provider $i$ if he reaches her threshold. Assume $\delta_{i}>0$, and hence $a_{i}=t_{i}=s_{i}+\delta_{i}$. We prove that provider $i$ can offer another commission schedule that brings her at least the same amount of revenue without discarding. 


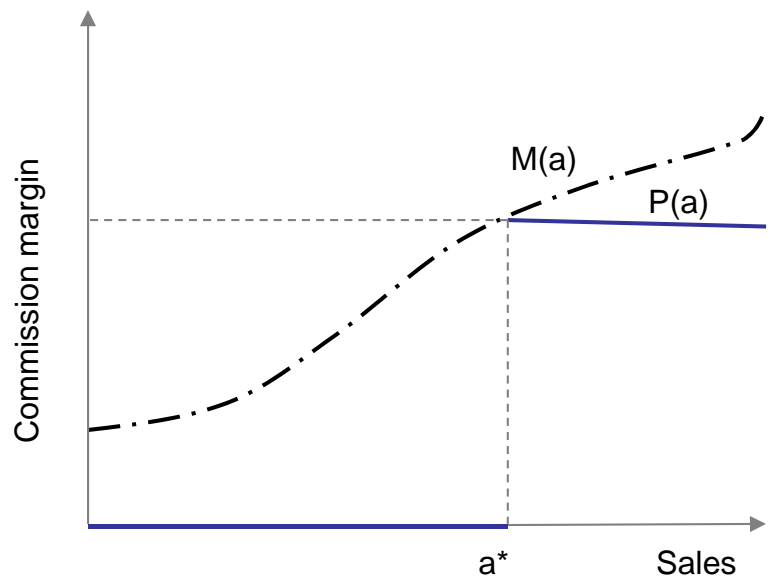

Figure 6 Converting a continuous margin schedule to a simple schedule

$$
\begin{aligned}
\pi_{i} & =\left(p_{i}-m_{i}\right)\left[s_{i}+\delta_{i}\right] \\
\pi_{R i} & =m_{i}\left[s_{i}+\delta_{i}\right]-p_{i} \delta_{i} \\
\pi_{R i} & \geq 0 \Rightarrow \delta_{i} \leq \frac{m_{i} s_{i}}{p_{i}-m_{i}}
\end{aligned}
$$

Now, provider $i$ can offer $m_{i}^{\prime}=p_{i}-\left(p_{i}-m_{i}\right) \frac{s_{i}+\delta_{i}}{s_{i}} \in\left[0, m_{i}\right)$ and $t_{i}^{\prime}=t_{i}-\delta_{i}$. Note that $a_{i}^{\prime}=t_{i}^{\prime}$ brings the same revenue for the provider $i$ and the broker as before. The broker does not choose a lower $a_{i}^{\prime}$ since it brings zero revenue for him, while $t_{i}^{\prime}$ brings a non-negative revenue. The broker may choose a higher $a_{i}^{\prime}$ which brings more revenue for the provider. Thus, the new strategy, which includes a lower commission margin and a lower threshold, dominates the previous strategy for provider $i$. In other words, there are multiple equilibria with the same profit split; and the provider always can choose the one that does not include discarding.

\section{B.2. Theorem 2}

Proof Notice that the providers do not go through a commission war up to their whole prices, because they can guarantee some sales even if they choose a lower commission. Each provider's commission margin is bounded by:

$$
p_{i}\left(d-b_{j}\right) \leq\left(p_{i}-m_{i}\right) b_{i} \Rightarrow m_{i} \leq \frac{b_{1}+b_{2}-d}{b_{i}} p_{i}
$$

Since each provider's margin needs to increase just to be higher than the other provider's margin, the cap of margins is the minimum of two caps.

$$
\frac{b_{2}}{p_{2}} \leq \frac{b_{1}}{p_{1}} \Rightarrow m_{i} \leq \frac{b_{1}+b_{2}-d}{b_{1}} p_{1}
$$

It means that provider 2 never chooses a margin in the interval $\left(\frac{b_{1}+b_{2}-d}{b_{1}} p_{1}, \frac{b_{1}+b_{2}-d}{b_{2}} p_{2}\right]$, because they are dominated by $\frac{b_{1}+b_{2}-d}{b_{1}} p_{1}$ which is high enough to beat provider 1 . 
In this case, there does not exist any pure-strategy Nash equilibrium, as both players' commissions adjust up to $\frac{b_{1}+b_{2}-d}{b_{1}} p_{1}$, and then the first provider jumps down to 0 , resulting in the secondary one also decrease her commission margin. Therefore, only a mixed-strategy equilibrium exists. Since the providers should be indifferent among all strategies in the support set of a mixed strategy, the revenues in the support set should be equal and we have:

Provider $j$ should be indifferent in each level $m \in\left[0, \frac{b_{1}+b_{2}-d}{b_{1}} p_{1}\right]$ :

$$
\begin{aligned}
& P\left(m_{i}^{o} \leq m\right)\left(p_{j}-m\right) b_{j}+P\left(m_{i}^{o}>m\right)\left(p_{j}-m\right)\left(d-b_{i}\right)=\left(p_{j}-\frac{b_{1}+b_{2}-d}{b_{1}} p_{1}\right) b_{j} \\
& F_{i}(m)\left(p_{j}-m\right) b_{j}+\left(1-F_{i}(m)\right)\left(p_{j}-m\right)\left(d-b_{i}\right)=\left[b_{i} p_{j}-b_{j} p_{i}\right]^{+\frac{b_{1}+b_{2}-d}{b_{i}}}+p_{j}\left(d-b_{i}\right) \\
& F_{i}(m)=\left(\frac{1}{p_{j}-m}\right)\left[\frac{\left(p_{j} b_{i}-p_{i} b_{j}\right)^{+}}{b_{i}}+\frac{m\left(d-b_{i}\right)}{b_{1}+b_{2}-d}\right] \\
& \begin{aligned}
& P\left(m_{1}<m_{2}\right) \\
= & \int_{0}^{\frac{b_{1}+b_{2}-d}{b_{1}} p_{1}} F_{1}(m) f_{2}(m) d m
\end{aligned} \\
& =\int_{0}^{\frac{b_{1}+b_{2}-d}{b_{1}} p_{1}} \frac{1}{p_{2}-m}\left(\frac{p_{2} b_{1}-p_{1} b_{2}}{b_{1}}+\frac{m\left(d-b_{1}\right)}{b_{1}+b_{2}-d}\right) \frac{d-b_{2}}{b_{1}+b_{2}-d} \frac{p_{1}}{\left(p_{1}-m\right)^{2}} d m \\
& =\int_{0}^{\frac{b_{1}+b_{2}-d}{b_{1}} p_{1}} \frac{1}{p_{2}-m}\left(\frac{p_{2} b_{1}-p_{1} b_{2}}{b_{1}}+\frac{m\left(d-b_{1}\right)}{b_{1}+b_{2}-d}\right) \frac{d-b_{2}}{b_{1}+b_{2}-d} \frac{p_{1}}{\left(p_{1}-m\right)^{2}} d m \\
& =\int_{0}^{\frac{b_{1}+b_{2}-d}{b_{1}} p_{1}} \frac{1}{p_{2}-m} \frac{p_{2} b_{1}-p_{1} b_{2}}{b_{1}} \frac{d-b_{2}}{b_{1}+b_{2}-d} \frac{p_{1}}{\left(p_{1}-m\right)^{2}} d m \\
& +\int_{0}^{\frac{b_{1}+b_{2}-d}{b_{1}} p_{1}} \frac{1}{p_{2}-m} \frac{m\left(d-b_{1}\right)}{b_{1}+b_{2}-d} \frac{d-b_{2}}{b_{1}+b_{2}-d} \frac{p_{1}}{\left(p_{1}-m\right)^{2}} d m \\
& =\frac{p_{2} b_{1}-p_{1} b_{2}}{b_{1}} \frac{\left(d-b_{2}\right) p_{1}}{b_{1}+b_{2}-d} \int_{0}^{\frac{b_{1}+b_{2}-d}{b_{1}}} p_{1} \frac{1}{\left(p_{2}-m\right)\left(p_{1}-m\right)^{2}} d m \\
& +\frac{\left(d-b_{1}\right) p_{1}}{b_{1}+b_{2}-d} \frac{d-b_{2}}{b_{1}+b_{2}-d} \int_{0}^{\frac{b_{1}+b_{2}-d}{b_{1}} p_{1}} \frac{m}{\left(p_{2}-m\right)\left(p_{1}-m\right)^{2}} d m \\
& =\left.\frac{\left(p_{2} b_{1}-p_{1} b_{2}\right)\left(d-b_{2}\right) p_{1}}{b_{1}\left(b_{1}+b_{2}-d\right)} \frac{p_{2}-p_{1}+\left(p_{1}-m\right) \ln \left(\frac{p_{1}-m}{p_{2}-m}\right)}{\left(p_{1}-p_{2}\right)^{2}\left(p_{1}-m\right)}\right|_{0} ^{\frac{b_{1}+b_{2}-d}{b_{1}}} p_{1} \\
& +\left.\frac{\left(d-b_{1}\right)\left(d-b_{2}\right) p_{1}}{\left(b_{1}+b_{2}-d\right)^{2}} \frac{p_{1}\left(p_{2}-p_{1}\right)+p_{2}\left(p_{1}-m\right) \ln \left(\frac{p_{1}-m}{p_{2}-m}\right)}{\left(p_{1}-p_{2}\right)^{2}\left(p_{1}-m\right)}\right|_{0} ^{\frac{b_{1}+b_{2}-d}{b_{1}} p_{1}} \\
& =\frac{\left(p_{2} b_{1}-p_{1} b_{2}\right)\left(d-b_{2}\right) p_{1}}{b_{1}\left(b_{1}+b_{2}-d\right)}\left[\frac{p_{2}-p_{1}+\frac{d-b_{2}}{b_{1}} p_{1} \ln \left(\frac{\left(d-b_{2}\right) p_{1}}{b_{1} p_{2}-\left(b_{1}+b_{2}-d\right) p_{1}}\right) b_{1}}{\left(p_{1}-p_{2}\right)^{2}\left(d-b_{2}\right) p_{1}}-\frac{p_{2}-p_{1}+p_{1} \ln \left(\frac{p_{1}}{p_{2}}\right)}{\left(p_{1}-p_{2}\right)^{2} p_{1}}\right] \\
& +\frac{\left(d-b_{1}\right)\left(d-b_{2}\right) p_{1}}{\left(b_{1}+b_{2}-d\right)^{2}}\left[\frac{p_{1}\left(p_{2}-p_{1}\right)+p_{2} \frac{d-b_{2}}{b_{1}} p_{1} \ln \left(\frac{\left(d-b_{2}\right) p_{1}}{b_{1} p_{2}-\left(b_{1}+b_{2}-d\right) p_{1}}\right) b_{1}}{\left(p_{1}-p_{2}\right)^{2}\left(d-b_{2}\right) p_{1}}-\frac{p_{1}\left(p_{2}-p_{1}\right)+p_{2} p_{1} \ln \left(\frac{p_{1}}{p_{2}}\right)}{\left(p_{1}-p_{2}\right)^{2} p_{1}}\right] \\
& =\frac{\left(p_{2} b_{1}-p_{1} b_{2}\right)\left(d-b_{2}\right) p_{1}}{b_{1}\left(b_{1}+b_{2}-d\right)}\left[\frac{\left(p_{2}-p_{1}\right) b_{1}+\frac{d-b_{2}}{b_{1}} p_{1} \ln \left(\frac{\left(d-b_{2}\right) p_{1}}{b_{1} p_{2}-\left(b_{1}+b_{2}-d\right) p_{1}}\right) b_{1}}{\left(p_{1}-p_{2}\right)^{2}\left(d-b_{2}\right) p_{1}}-\frac{p_{2}-p_{1}+p_{1} \ln \left(\frac{p_{1}}{p_{2}}\right)}{\left(p_{1}-p_{2}\right)^{2} p_{1}}\right] \\
& +\frac{\left(d-b_{1}\right)\left(d-b_{2}\right) p_{1}}{\left(b_{1}+b_{2}-d\right)^{2}}\left[\frac{p_{1}\left(p_{2}-p_{1}\right) b_{1}+p_{2} \frac{d-b_{2}}{b_{1}} p_{1} \ln \left(\frac{\left(d-b_{2}\right) p_{1}}{b_{1} p_{2}-\left(b_{1}+b_{2}-d\right) p_{1}}\right) b_{1}}{\left(p_{1}-p_{2}\right)^{2}\left(d-b_{2}\right) p_{1}}-\frac{p_{1}\left(p_{2}-p_{1}\right)+p_{2} p_{1} \ln \left(\frac{p_{1}}{p_{2}}\right)}{\left(p_{1}-p_{2}\right)^{2} p_{1}}\right] \\
& =\frac{\left(p_{2} b_{1}-p_{1} b_{2}\right)\left(d-b_{2}\right) p_{1}}{b_{1}\left(b_{1}+b_{2}-d\right)}\left[\frac{b_{1}+b_{2}-d}{\left(p_{2}-p_{1}\right)\left(d-b_{2}\right) p_{1}}-\frac{\ln \left(\frac{\left(d-b_{2}\right) p_{2}}{b_{1} p_{2}-\left(b_{1}+b_{2}-d\right) p_{1}}\right)}{\left(p_{1}-p_{2}\right)^{2}}\right]
\end{aligned}
$$




$$
\begin{aligned}
& +\frac{\left(d-b_{1}\right)\left(d-b_{2}\right) p_{1}}{\left(b_{1}+b_{2}-d\right)^{2}}\left[\frac{b_{1}+b_{2}-d}{\left(p_{2}-p_{1}\right)\left(d-b_{2}\right)}-\frac{p_{2} \ln \left(\frac{\left(d-b_{2}\right) p_{2}}{b_{1} p_{2}-\left(b_{1}+b_{2}-d\right) p_{1}}\right)}{\left(p_{1}-p_{2}\right)^{2}}\right] \\
& =\frac{p_{2} b_{1}-p_{1} b_{2}}{b_{1}\left(p_{2}-p_{1}\right)}+\frac{\left(d-b_{1}\right) p_{1}}{\left(b_{1}+b_{2}-d\right)\left(p_{2}-p_{1}\right)} \\
& \left.-\left[\frac{\left(d-b_{1}\right)\left(d-b_{2}\right) p_{1} p_{2}}{\left(b_{1}+b_{2}-d\right)^{2}}+\frac{\left(p_{2} b_{1}-p_{1} b_{2}\right)\left(d-b_{2}\right) p_{1}}{b_{1}\left(b_{1}+b_{2}-d\right)}\right] \frac{\ln \left(\frac{\left(d-b_{2}\right) p_{2}}{b_{1} p_{2}-\left(b_{1}+b_{2}-d\right) p_{1}}\right)}{\left(p_{1}-p_{2}\right)^{2}}\right]
\end{aligned}
$$

Total revenue of the chain is equal to:

$$
\begin{aligned}
& \pi_{\text {chain }}=P\left(m_{1}^{o}<m_{2}^{o}\right)\left(p_{1} r_{1}+p_{2} b_{2}\right)+P\left(m_{2}^{o}<m_{1}^{o}\right)\left(p_{1} b_{1}+p_{2} r_{2}\right) \\
&=p_{1} b_{1}+p_{2}\left(d-b_{1}\right)+\left(b_{1}+b_{2}-d\right)\left(p_{2}-p_{1}\right) P\left(m_{1}<m_{2}\right) \\
&=p_{1} b_{1}+p_{2}\left(d-b_{1}\right)+\frac{\left(p_{2} b_{1}-p_{1} b_{2}\right)\left(b_{1}+b_{2}-d\right)}{b_{1}}+\left(d-b_{1}\right) p_{1} \\
&-\left[\frac{\left(d-b_{1}\right)\left(d-b_{2}\right) p_{1} p_{2}}{\left(b_{1}+b_{2}-d\right)}+\frac{\left(p_{2} b_{1}-p_{1} b_{2}\right)\left(d-b_{2}\right) p_{1}}{b_{1}}\right] \frac{\ln \left(\frac{\left(d-b_{2}\right) p_{2}}{b_{1} p_{2}-\left(b_{1}+b_{2}-d\right) p_{1}}\right)}{p_{1}-p_{2}} \\
&=p_{1} d+p_{2} b_{2}-\frac{p_{1} b_{2}\left(b_{1}+b_{2}-d\right)}{b_{1}} \\
&-\frac{\left(d-b_{2}\right) p_{1}}{p_{1}-p_{2}}\left[\frac{\left(d-b_{1}\right) p_{2}}{\left(b_{1}+b_{2}-d\right)}+\frac{\left(p_{2} b_{1}-p_{1} b_{2}\right)}{b_{1}}\right] \ln \left(\frac{\left(d-b_{2}\right) p_{2}}{b_{1} p_{2}-\left(b_{1}+b_{2}-d\right) p_{1}}\right) \\
& \pi_{B}=\pi_{\text {chain }}-\pi_{1}-\pi_{2}\left(d-b_{2}\right) p_{1} \\
&\left.=p_{1} b_{2}-\frac{\left(d-b_{1}\right) p_{2}}{p_{1}-p_{2}}+\frac{\left(p_{2} b_{1}-p_{1} b_{2}\right)}{b_{1}}\right] \ln \left(\frac{\left(d-b_{2}\right) p_{2}}{b_{1} p_{2}-\left(b_{1}+b_{2}-d\right) p_{1}}\right)
\end{aligned}
$$

If $p_{1}=p_{2}=p$ then the phrase $\frac{1}{p_{1}-p_{2}} \ln \left(\frac{\left(d-b_{2}\right) p_{2}}{b_{1} p_{2}-\left(b_{1}+b_{2}-d\right) p_{1}}\right)$ is undefined. We find the limit, using t'Hospital's rule, which results in:

$$
\pi_{B}=p \frac{b_{2}}{b_{1}}\left(b_{1}+b_{2}-d\right)
$$

\section{B.3. Theorem 3}

Proof Notice that if $\left(t_{1}^{*}, m_{1}^{*}, t_{2}^{*}, m_{2}^{*}\right)$ is a Nash equilibrium, then $\left(t_{1}^{*}, t_{2}^{*}\right)$ should be a Nash equilibrium for fixed $\left(m_{1}^{*}, m_{2}^{*}\right)$. Hence we use Theorem 7 . Note that in every case, we deal with a competitive market, since if market is not competitive, the provider without ample capacity has incentive to decrease her $m$.

- The primary provider, provider $i$, sells $b_{i}$. In other words, for the primary provider $a_{i}^{*}=\min \left[c_{i}, \hat{s}_{i}+\right.$ $\left.\frac{\hat{\pi}_{i}-\hat{\pi}_{j}}{p_{i}-m_{i}}\right]=b_{i}$. Note that in Theorem 1 , we proved that there is no discarding.

- $\hat{\pi}_{1}=\hat{\pi}_{2}$. Otherwise it's optimal for the primary provider to decrease her commission margin and increase her revenue. Notice that if, for example, $\hat{\pi}_{j}<\hat{\pi}_{i}$ then provider $i$ 's revenue is $\left(p_{i}-m_{i}\right) b_{i}$ and can be increased by decreasing $m_{i}$. When the two providers have equal commission fees, the broker will choose one of them as the preferred provider. 
- $\left(p_{i}-m_{i}\right) b_{i}=p_{i}\left(d-b_{j}\right)$ for $i=1,2$. Since $\hat{\pi}_{1}=\hat{\pi}_{2}$, then broker is indifferent between choosing any of the providers as the primary one. Since the primary provider, provider $i$, has no incentive to change her actions to become secondary we have $\left(p_{i}-m_{i}\right) b_{i} \geq p_{i}\left(d-b_{j}\right)$. And, since provider $j$ doe not have any incentive to become the primary provider, and hence we have $\left(p_{j}-m_{j}\right) b_{j} \leq p_{j}\left(d-b_{i}\right)$. Note that these inequalities hold for $i=1,2$ and gives rise to the desired equalities.

- $m_{i}^{*}=\frac{b_{i}+b_{j}-d}{b_{i}} p$ for $i=1,2$.

Table 3 provides results od sensitivity analysis based on Theorem 3 .

As the demand increases:

- $\frac{\partial \pi_{i}}{\partial d} \in\left[0, p_{i}\right]$

- $\frac{\partial \pi_{B}}{\partial d} \in\left[-\min \left[p_{1}, p_{2}\right], \min \left[p_{1}, p_{2}\right]\right]$

As the broker's power increases:

- $\frac{\partial \pi_{i}}{\partial d_{0}} \in\left[-\max \left[\min \left[p_{1}, p_{2}\right],\left(p_{i}-p_{j}\right)^{+}\right],\left(p_{i}-p_{j}\right)^{+}\right]$

- $\frac{\partial \pi_{B}}{\partial d_{0}} \in\left[0, \min \left[p_{1}, p_{2}\right]\right]$

Table 2 Sensitivity Analysis

\begin{tabular}{|l|c|c|c|c|}
\hline & $\frac{\partial \pi_{i}}{\partial d}$ & $\frac{\partial \pi_{B}}{\partial d}$ & $\frac{\partial \pi_{i}}{\partial d_{0}}$ & $\frac{\partial \pi_{B}}{\partial d_{0}}$ \\
\hline$c_{1} \leq d_{0}+d_{1}$ & $\min \left[p_{1}, p_{2}\right]$ & $-\min \left[p_{1}, p_{2}\right]$ & 0 & 0 \\
$c_{2} \leq d_{0}+d_{2}$ & & & & \\
\hline$c_{1}>d_{0}+d_{1}$ & $\frac{d_{i}}{d} p_{i}+\frac{d_{0}}{d}\left(p_{i}-p_{j}\right)^{+}$ & $\frac{d_{0}}{d} \min \left[p_{1}, p_{2}\right]$ & $-p_{i}[0,1]+\left(p_{i}-p_{j}\right)^{+}$ & $\min \left[p_{1}, p_{2}\right]$ \\
$c_{2}>d_{0}+d_{2}$ & & & & \\
\hline$c_{i} \leq d_{0}+d_{i}$ & $\frac{d_{i}}{d} \min \left[p_{1}, p_{2}\right]$ & $\frac{d_{i}}{d} \min \left[p_{1}, p_{2}\right]$ & {$\left[-\min \left[p_{i}, p_{j}\right], 0\right]$} & {$\left[0, \min \left[p_{1}, p_{2}\right]\right]$} \\
$c_{j}>d_{0}+d_{j}$ & & & & {$\left[0, \min \left[p_{1}, p_{2}\right]\right]$} \\
\hline$c_{i}>d_{0}+d_{i}$ & $p_{i}-\frac{d_{i}}{d}\left(p_{i}-p_{j}\right)^{+}$ & $\frac{d_{i}}{d} \min \left[p_{1}, p_{2}\right]$ & {$\left[-\left(p_{i}-p_{j}\right)^{+}, 0\right]$} & \\
$c_{j} \leq d_{0}+d_{j}$ & & & & \\
\hline
\end{tabular}

\section{B.4. Theorem 4}

Proof If provider $i$ sells directly to loyal customers, she collects all the revenue from them which equals $p_{i} d_{i}$. To analyze the revenue from selling through the broker, it should be noticed that the total demand which is sold through the broker has been reduced to $d-d_{i}$. Thus, based on Theorem 3 , the revenue of provider $i$ from the broker's sales is $p_{i}\left(\left(d-d_{i}\right)-b_{j}\right)+\left(p_{i}-p_{j}\right)^{+}\left(\left(b_{i}-d_{i}\right)+b_{j}-\left(d-d_{i}\right)\right)$. It means that:

$$
\begin{aligned}
\pi_{i}^{d} & =p_{i} d_{i}+p_{i}\left(d-d_{i}-b_{j}\right)+\left(p_{i}-p_{j}\right)^{+}\left(b_{i}+b_{j}-d\right) \\
& =p_{i}\left(d-b_{j}\right)+\left(p_{i}-p_{j}\right)^{+}\left(b_{i}+b_{j}-d\right) \\
& =\pi_{i}^{*}
\end{aligned}
$$

\section{B.5. Theorem 5}

Proof The providers' revenue comparison before and after thresholds have been done in Table 4, assuming $b_{1}>b_{2}$. If $b_{1}=b_{2}$, the providers' revenues do not change. 
Table 3 Strategic Effects

\begin{tabular}{|l|c|c|c|c|}
\hline & & Before Thresholds $\left(\pi^{o}\right)$ & After Thresholds $\left(\pi^{*}\right)$ & Comparison \\
\hline$p_{1} \leq p_{2}$ & 1 & $p_{1}\left(d-b_{2}\right)$ & $p_{1}\left(d-b_{2}\right)$ & Unchanged \\
& 2 & $p_{2}\left(d-b_{1}\right)+\left(p_{2}-\frac{b_{2}}{b_{1}} p_{1}\right)\left(b_{1}+b_{2}-d\right)$ & $p_{2}\left(d-b_{1}\right)+\left(p_{2}-p_{1}\right)\left(b_{1}+b_{2}-d\right)$ & Lost \\
\hline$p_{1}>p_{2}$ & 1 & $p_{1}\left(d-b_{2}\right)$ & $p_{1}\left(d-b_{2}\right)+\left(p_{1}-p_{2}\right)\left(b_{1}+b_{2}-d\right)$ & Win \\
$\frac{b_{1}}{p_{1}}>\frac{b_{2}}{p_{2}}$ & 2 & $p_{2}\left(d-b_{1}\right)+\left(p_{2}-\frac{b_{2}}{b_{1}} p_{1}\right)\left(b_{1}+b_{2}-d\right)$ & $p_{2}\left(d-b_{1}\right)$ & Lost \\
\hline$p_{1}>p_{2}$ & 1 & $p_{1}\left(d-b_{2}\right)+\left(p_{1}-\frac{b_{1}}{b_{2}} p_{2}\right)\left(b_{1}+b_{2}-d\right)$ & $p_{1}\left(d-b_{2}\right)+\left(p_{1}-p_{2}\right)\left(b_{1}+b_{2}-d\right)$ & Win \\
$\frac{b_{1}}{p_{1}} \leq \frac{b_{2}}{p_{2}}$ & 2 & $p_{2}\left(d-b_{1}\right)$ & $p_{2}\left(d-b_{1}\right)$ & Unchanged \\
\hline
\end{tabular}

